\title{
ON THE ALMOST SURE RUNNING MAXIMA OF SOLUTIONS OF AFFINE STOCHASTIC FUNCTIONAL DIFFERENTIAL EQUATIONS*
}

\author{
JOHN A. D. APPLEBY ${ }^{\dagger}$, XUERONG MAO ${ }^{\ddagger}$, AND HUIZHONG WU§
}

\begin{abstract}
This paper studies the large fluctuations of solutions of scalar and finite-dimensional affine stochastic functional differential equations with finite memory as well as related nonlinear equations. We find conditions under which the exact almost sure growth rate of the running maximum of each component of the system can be determined, both for affine and nonlinear equations. The proofs exploit the fact that an exponentially decaying fundamental solution of the underlying deterministic equation is sufficient to ensure that the solution of the affine equation converges to a stationary Gaussian process.
\end{abstract}

Key words. stochastic functional differential equation, Gaussian process, stationary process, differential resolvent, running maxima, almost sure asymptotic estimation, finite delay

AMS subject classifications. 34K06, 34K25, 34K50, 34K60, 60F 15, 60F 10

DOI. $10.1137 / 080738404$

1. Introduction. Increasingly real-world systems are modelled using stochastic differential equations with delay, as they represent systems which evolve in a random environment and whose evolution depends on the past states of the system through either memory or time delay. Examples include population biology (Mao [41], Mao and Rassias [42, 43]), neural networks (Blythe, Mao, and Shah [10]), viscoelastic materials subjected to heat or mechanical stress (Drozdov and Kolmanovskiı̌ [27], Caraballo et al. [17], Mizel and Trutzer [46, 47]), or financial mathematics (Anh and Inoue [1], Ahn, Inoue, and Kasahara [2], Arriojas et al. [9], Hobson and Rogers [30], Bouchaud and Cont [12]).

In such stochastic models of phenomena in engineering and physics it is often of great importance to know that the system is stable in the sense that the solution of the mathematical model converges in some sense to equilibrium. Consequently, a great deal of mathematical activity has been devoted to the question of stability of point equilibria of stochastic functional differential equations and also to the rate at which solutions converge. The literature is extensive, but a flavor of the work can be found in the monographs of Mao [38, 39], Mohammed [48], and Kolmanovskii and Myshkis [33].

However, in disciplines such as mathematical biology or finance, it is less usual for systems to converge to an equilibrium; more typically, the solutions may be stable

*Received by the editors October 17, 2008; accepted for publication (in revised form) December 21, 2009; published electronically March 31, 2010. This work was supported by Science Foundation Ireland (SFI) under Research Frontiers Programme grant RFP/MAT/0018 "Stochastic Functional Differential Equations with Long Memory."

http://www.siam.org/journals/sima/42-2/73840.html

$\dagger$ Edgeworth Centre for Financial Mathematics, School of Mathematical Sciences, Dublin City University, Glasnevin, Dublin 9, Ireland (john.appleby@dcu.ie). This author's research was supported by SFI under Mathematics Initiative 2007 grant 07/MI/008 "Edgeworth Centre for Financial Mathematics."

${ }^{\ddagger}$ Department of Statistical and Modelling Science, University of Strathclyde, Livingstone Tower, 26 Richmond Street, Glasgow G1 1XT, United Kingdom (xuerong@stams.strath.ac.uk).

$\S$ School of Mathematical Sciences, Dublin City University, Glasnevin, Dublin 9, Ireland (huizhong. wu4@mail.dcu.ie). 
in the sense that there is a stationary distribution to which the solution converges (see, e.g., Reiss, Riedle, and van Gaans [53], Küchler and Mensch [34], Mao [40]) but that the solution is unbounded in the sense that the running maximum $X^{*}(t):=$ $\sup _{0 \leq s \leq t}|X(s)|$ obeys

$$
\lim _{t \rightarrow \infty} X^{*}(t)=\infty, \quad \text { with (at least) positive probability. }
$$

Therefore, it is natural to ask at what rate the running maxima tend to infinity or, more precisely, to find a deterministic function $\rho$ with $\rho(t) \rightarrow \infty$ as $t \rightarrow \infty$ such that

$$
\lim _{t \rightarrow \infty} \frac{X^{*}(t)}{\rho(t)}=1 \quad \text { a.s. }
$$

We call such a function $\rho$ the essential growth rate of the running maxima of $X$. In applications this is important, as the size of the large fluctuations may represent the largest bubble or crash in a financial market, the largest epidemic in a disease model, or a population explosion in an ecological model.

Despite the importance of this problem, to date there is comparatively little literature regarding the size of such large fluctuations and, to the best of our knowledge, no comprehensive theory for linear stochastic functional differential equations. Despite this, Mao and Rassias [43] have established upper bounds on the essential growth rate of the running maxima of solutions of some special stochastic delay differential equations (SDDEs) with fixed delays, with their results having particular application to population biology. Their methods enable them to recover results for highly nonlinear systems which are, moreover, sharp in the sense that the rate of growth of the corresponding nondelay systems is recovered when the fixed delay is set equal to zero. However, their methods do not automatically extend to differential equations with more general delay functionals, nor can they obtain lower bounds on the essential rate of growth of the running maxima.

This paper deals with a simpler class of stochastic functional differential equations (SFDEs) than [43] (in the sense that the equations are essentially linear) but with a more general type of delay functional, covering both point and distributed delays by using measures in the delay. In common with [43], but by different methods, we obtain an upper bound on the rate of growth of the running maxima. However, in contrast to [43], we are also able to establish a lower bound on the rate of growth of the running maxima; indeed, as these bounds are equal, we can determine the exact almost sure rate of growth of the running maxima. The results exploit the fact that given an exponentially decaying resolvent, the finite delay in the equation forces the limiting autocovariance function to decay exponentially fast, so that the solution of the linear equation is an asymptotically stationary Gaussian process. The results apply to both scalar and finite-dimensional equations and can, moreover, be extended to equations with a weak nonlinearity at infinity.

More precisely, we study the asymptotic behavior of the finite-dimensional process which satisfies

$$
\begin{array}{r}
X(t)=\psi(0)+\int_{0}^{t} L\left(X_{s}\right) d s+\int_{0}^{t} \Sigma d B(s), \quad t \geq 0, \\
X(t)=\phi(t), \quad t \in[-\tau, 0],
\end{array}
$$

Copyright (c) by SIAM. Unauthorized reproduction of this article is prohibited. 
where $B$ is an $m$-dimensional standard Brownian motion, $\Sigma$ is a $d \times m$ matrix with real entries, and $L: C[-\tau, 0] \rightarrow \mathbb{R}^{d}$ is a linear functional with $\tau \geq 0$ and

$$
L(\phi)=\int_{[-\tau, 0]} \nu(d s) \phi(s), \quad \phi \in C\left([-\tau, 0] ; \mathbb{R}^{d}\right) .
$$

The asymptotic behavior of (1.2)-(1.3) is determined in the case when the resolvent $r$ of the deterministic equation $x^{\prime}(t)=L\left(x_{t}\right), t \geq 0$, obeys $r \in L^{1}\left([0, \infty) ; \mathbb{R}^{d \times d}\right)$. In particular, we show that the running maxima of each component grow according to

$$
\limsup _{t \rightarrow \infty} \frac{\left\langle X(t), \mathbf{e}_{i}\right\rangle}{\sqrt{2 \log t}}=\sigma_{i}, \quad \liminf _{t \rightarrow \infty} \frac{\left\langle X(t), \mathbf{e}_{i}\right\rangle}{\sqrt{2 \log t}}=-\sigma_{i} \quad \text { a.s. }
$$

where $\sigma_{i}>0$ depends on $\Sigma$ and the resolvent $r$. Moreover,

$$
\limsup _{t \rightarrow \infty} \frac{|X(t)|_{\infty}}{\sqrt{2 \log t}}=\max _{i=1, \ldots, d} \sigma_{i} \quad \text { a.s. }
$$

We can also subject (1.2)-(1.3) to a general nonlinear perturbation to get the equation

$$
d X(t)=\left(L\left(X_{t}\right)+N\left(t, X_{t}\right)\right) d t+\Sigma d B(t), \quad t \geq 0,
$$

and still retain the asymptotic behavior of (1.2)-(1.3). More specifically, if the nonlinear functional $N:[0, \infty) \times C[-\tau, 0] \rightarrow \mathbb{R}^{d}$ is of smaller than linear order as $\|\varphi\|_{2}:=\sup _{-\tau \leq s \leq 0}|\varphi(s)|_{2} \rightarrow \infty$ in the sense that

$$
\lim _{\|\varphi\|_{2} \rightarrow \infty} \frac{\left|N\left(t, \phi_{t}\right)\right|_{2}}{\|\varphi\|_{2}}=0 \text { uniformly in } t \geq 0,
$$

then (1.4) and (1.5) still hold.

Linear stochastic delay difference equations are commonly seen in the time series modelling of interest rates and volatilities in inefficient markets, in which historical information is incorporated in the dynamical system at any given time. An autoregressive (AR) model can be seen as a discretized version of the linear SFDE (1.2)-(1.3) when the measure $\nu$ is purely discrete. More precisely, if the continuous-time equation has only an instantaneous term and $p$ point delays equally spaced in time, an $\operatorname{AR}(p)$ process results from the discretization. If the mesh size of the discretization is chosen sufficiently small, properties such as stationarity of the continuous equation can be preserved by the AR model. Conversely, an appropriately parameterized $\operatorname{AR}(p)$ model can converge weakly to the solution of (1.2)-(1.3) with a discrete measure as the parameter tends to a limit.

An extension and application in which the conditional variance obeys an AR equation are given by the generalized autoregressive conditional heteroskedasticity (GARCH) model developed by Bollerslev (cf., e.g., [11, 25]); such models are often used to model stock volatilities. There is an extensive literature on GARCH and AR models applied to finance, with nice recent introductions provided in, e.g., [28]. A wealth of basic results on linear time series models is also contained in the classic text [14]. The results in this paper concerning Gaussian stationary solutions of linear SFDEs provide the basic framework for estimating the large deviations of interest rates or volatilities simulated by continuous-time semimartingale analogues of both scalar and vector AR processes. An interesting and related literature on continuous-time 
linear stochastic models also exists in the time series literature (see, e.g., [13, 15, 45]), but the emphasis in those works does not overlap with the thrust of this paper.

The nonlinear problem (1.6) studied in this paper deals only with nonlinearity that is lower than linear order in the space variable at infinity in a sense made precise by (1.7). It is therefore interesting to ask how the results here could be developed to deal with other forms of nonlinearity in the presence of additive noise. In [8] the asymptotic behavior of scalar SFDEs of the form

$$
d X(t)=\left(a X(t)+b \sup _{t-\tau \leq s \leq t} X(s)\right) d t+\sigma d B(t), \quad t \geq 0
$$

is considered. Note that (1.8) is not in the form of either (1.2)-(1.3) or (1.6) with the condition (1.7). In [8] it is shown that if the solution is recurrent on the real line, then the presence of the maximum functional does not significantly change the essential growth rate of the solution of the related nondelay linear equation $d Y(t)=$ $\alpha Y(t) d t+\sigma d B(t)$, where $\alpha<0$. More specifically, it is shown that there exist deterministic $c_{1}, c_{2}$ such that

$$
0<c_{1} \leq \limsup _{t \rightarrow \infty} \frac{|X(t)|}{\sqrt{2 \log t} \leq c_{2}<+\infty \quad \text { a.s. }}
$$

which recovers the exact square root logarithmic growth rate of $Y$

$$
\limsup _{t \rightarrow \infty} \frac{|Y(t)|}{\sqrt{2 \log t}}=\frac{|\sigma|}{\sqrt{2|\alpha|}} \text { a.s. }
$$

Since we demonstrate in the present paper that equations of the form (1.6) have an exact square root logarithmic growth rate, this suggests that it is linearity, or "near linearity," that generates Gaussian-like large fluctuations.

For a scalar autonomous SDE which has no delay and whose solution is stationary we can apply Motoo's theorem (cf. $[50,31])$ to estimate the growth rate of the running maximum, even when the drift coefficient is not of linear leading order at infinity (in contrast to (1.6) and (1.8) with the condition (1.7)). These techniques can even be extended to finite-dimensional and nonstationary processes (see, e.g., [7]). Similarly, if some delay terms are introduced into a stationary nonlinear SDE, provided the order of this delay term is smaller than that of the instantaneous term at infinity, we show in [5] that the size of the large fluctuations of the nondelay process is preserved. The essential growth rate in this case depends on the degree of nonlinearity of the instantaneous term. In contrast to the present paper, however, results obtained in [5] do not exactly characterize the essential growth rate in the sense of (1.1) or (1.5); rather results of the form

$$
0<c_{1} \leq \limsup _{t \rightarrow \infty} \frac{X^{*}(t)}{\rho(t)} \leq c_{2}<+\infty \quad \text { a.s. }
$$

are established for constants $c_{1}, c_{2}$ and the appropriate increasing function $\rho$.

This paper is organized as follows. Section 2 gives the background material on SFDEs and introduces notation used. The main results of the paper are listed and discussed in section 3. The proof of (1.5) in the scalar case is given in section 4.1. The proof of (1.4) and (1.5) for the finite-dimensional equation is given in section 4.2, with the corresponding results for the nonlinear equation being presented in section 4.3. Finally, the proofs of auxiliary lemmata are given in section 5 . 
2. Preliminaries. Let $d, m$ be some positive integers and $\mathbb{R}^{d \times m}$ denote the space of all $d \times m$ matrices with real entries. We equip $\mathbb{R}^{d \times m}$ with a norm $|\cdot|$ and write $\mathbb{R}^{d}$ if $m=1$ and $\mathbb{R}$ if $d=m=1$. We denote by $\mathbb{R}^{+}$the half-line $[0, \infty)$. The complex plane is denoted by $\mathbb{C}$.

Let $M\left([-\tau, 0], \mathbb{R}^{d \times d}\right)$ be the space of finite signed Borel measures on $[-\tau, 0]$ with values in $\mathbb{R}^{d \times d}$. The total variation of a measure $\nu$ in $M\left([-\tau, 0], \mathbb{R}^{d \times d}\right)$ on a Borel set $B \subseteq[-\tau, 0]$ is defined by

$$
|\nu|(B):=\sup \sum_{i=1}^{N}\left|\nu\left(E_{i}\right)\right|
$$

where $\left(E_{i}\right)_{i=1}^{N}$ is a partition of $B$ and the supremum is taken over all partitions. The total variation defines a positive scalar measure $|\nu|$ in $M([-\tau, 0], \mathbb{R})$. If one specifies temporarily the norm $|\cdot|$ as the $l^{1}$-norm on the space of real-valued sequences and identifies $\mathbb{R}^{d \times d}$ by $\mathbb{R}^{d^{2}}$, one can easily establish for the measure $\nu=\left(\nu_{i, j}\right)_{i, j=1}^{d^{2}}$ the inequality

$$
|\nu|(B) \leq C \sum_{i=1}^{d} \sum_{j=1}^{d}\left|\nu_{i, j}\right|(B) \quad \text { for every Borel set } B \subseteq[-\tau, 0]
$$

with $C=1$. Then, by the equivalence of every norm on finite-dimensional spaces, the inequality (2.1) holds true for the arbitrary norms $|\cdot|$ and some constant $C>0$. Moreover, as in the scalar case we have the fundamental estimate

$$
\left|\int_{[-\tau, 0]} \nu(d s) f(s)\right| \leq \int_{[-\tau, 0]}|f(s)||\nu|(d s)
$$

for every function $f:[-\tau, 0] \rightarrow \mathbb{R}^{d \times d^{\prime}}$ which is $|\nu|$-integrable.

We first turn our attention to the deterministic delay equation underlying the SDE (1.2)-(1.3). For a fixed constant $\tau \geq 0$ we consider the deterministic linear delay differential equation

$$
\begin{aligned}
x^{\prime}(t) & =\int_{[-\tau, 0]} \nu(d u) x(t+u) \quad \text { for } t \geq 0, \\
x(t) & =\phi(t) \quad \text { for } t \in[-\tau, 0]
\end{aligned}
$$

for a measure $\nu \in M\left([-\tau, 0], \mathbb{R}^{d \times d}\right)$. The initial function $\phi$ is assumed to be in the space $C[-\tau, 0]:=\left\{\phi:[-\tau, 0] \rightarrow \mathbb{R}^{d}:\right.$ continuous $\}$. A function $x:[-\tau, \infty) \rightarrow \mathbb{R}^{d}$ is called a solution of $(2.2)$ if $x$ is continuous on $[-\tau, \infty)$, its restriction to $[0, \infty)$ is continuously differentiable, and $x$ satisfies the first and second identities of (2.2) for all $t \geq 0$ and $t \in[-\tau, 0]$, respectively. It is well known that for every $\phi \in C[-\tau, 0]$ the problem (2.2) admits a unique solution $x=x(\cdot, \phi)$.

The fundamental solution or resolvent of (2.2) is the unique locally absolutely continuous function $r:[0, \infty) \rightarrow \mathbb{R}^{d \times d}$ which satisfies

$$
r(t)=I_{d}+\int_{0}^{t} \int_{[\max \{-\tau,-s\}, 0]} \nu(d u) r(s+u) d s \quad \text { for } t \geq 0,
$$

where $I_{d}$ is the $d \times d$ identity matrix. It plays a role which is analogous to the fundamental system in linear ordinary differential equations and the Green function in partial differential equations. For later convenience we set $r(t)=0$ for $t \in[-\tau, 0)$. 
The solution $x(\cdot, \phi)$ of (2.2) for an arbitrary initial segment $\phi$ exists, is unique, and can be represented as

$$
x(t, \phi)=r(t) \phi(0)+\int_{-\tau}^{0} \int_{[-\tau, u]} r(t+s-u) \nu(d s) \phi(u) d u \quad \text { for } t \geq 0 ;
$$

cf. Diekmann et al. [26, Chapter I].

Define the function $h_{\nu}: \mathbb{C} \rightarrow \mathbb{C}$ by

$$
h_{\nu}(\lambda)=\operatorname{det}\left(\lambda I_{d}-\int_{[-\tau, 0]} e^{\lambda s} \nu(d s)\right),
$$

where $\operatorname{det}(A)$ signifies the determinant of a $d \times d$ matrix $A$. Define also the set

$$
\Lambda=\left\{\lambda \in \mathbb{C}: h_{\nu}(\lambda)=0\right\} .
$$

The function $h$ is analytic, and so the elements of $\Lambda$ are isolated. Define

$$
v_{0}(\nu):=\sup \left\{\Re(\lambda): h_{\nu}(\lambda)=0\right\},
$$

where $\Re(z)$ denotes the real part of a complex number $z$. Furthermore, the cardinality of $\Lambda^{\prime}:=\Lambda \cap\left\{\Re(\lambda)=v_{0}(\nu)\right\}$ is finite. Then there exists $\varepsilon_{0}>0$ such that for every $\varepsilon \in\left(0, \varepsilon_{0}\right)$ we have

$$
e^{-v_{0}(\nu) t} r(t)=\sum_{\lambda_{j} \in \Lambda^{\prime}}\left\{p_{j}(t) \cos \left(\Im\left(\lambda_{j}\right) t\right)+q_{j}(t) \sin \left(\Im\left(\lambda_{j}\right) t\right)\right\}+o\left(e^{-\varepsilon t}\right), \quad t \rightarrow \infty,
$$

where $p_{j}$ and $q_{j}$ are matrix-valued polynomials of degree $m_{j}-1$, with $m_{j}$ being the multiplicity of the zero $\lambda_{j} \in \Lambda^{\prime}$ of $h$, and $\Im(z)$ denoting the imaginary part of a complex number $z$. Hence, for every $\epsilon>0$ there exists a $C(\epsilon)>0$ such that

$$
|r(t)| \leq C(\epsilon) e^{\left(v_{0}(\nu)-\epsilon\right) t}, \quad t \geq 0 .
$$

Therefore if $v_{0}(\nu)<0$, then $r$ decays to zero exponentially. This is a simple restatement of Diekmann et al. [26, Theorem 1.5.4 and Corollary 1.5.5]. Furthermore, the following lemma regarding $r$ is given in [4].

Lemma 1. Let $r$ satisfy (2.3), and let $v_{0}(\nu)$ be defined as (2.5). Then the following statements are equivalent:

(a) $v_{0}(\nu)<0$.

(b) $r$ decays exponentially as $t \rightarrow \infty$.

(c) $r(t) \rightarrow 0$ as $t \rightarrow \infty$.

(d) $r \in L^{1}\left(\mathbb{R}^{+} ; \mathbb{R}^{d \times d}\right)$.

(e) $r \in L^{2}\left(\mathbb{R}^{+} ; \mathbb{R}^{d \times d}\right)$.

Let us introduce some notation for (2.2). For a function $x:[-\tau, \infty) \rightarrow \mathbb{R}^{d}$ we define the segment of $x$ at time $t \geq 0$ by the function

$$
x_{t}:[-\tau, 0] \rightarrow \mathbb{R}^{d}, \quad x_{t}(u):=x(t+u) .
$$

If we equip the space $C[-\tau, 0]$ of continuous functions with the supremum norm, the Riesz representation theorem guarantees that every continuous functional $L$ : $C[-\tau, 0] \rightarrow \mathbb{R}^{d}$ is of the form

$$
L(\psi)=\int_{[-\tau, 0]} \nu(d u) \psi(u)
$$

Copyright $@$ by SIAM. Unauthorized reproduction of this article is prohibited. 
for a $d \times d$ matrix-valued measure $\nu \in M([-\tau, 0], \mathbb{R})$. Hence, we will write $(2.2)$ in the form

$$
x^{\prime}(t)=L\left(x_{t}\right) \quad \text { for } t \geq 0, \quad x_{0}=\phi
$$

and assume $L$ to be a continuous and linear functional on $C\left([-\tau, 0] ; \mathbb{R}^{d}\right)$.

Let us fix a complete probability space $(\Omega, \mathcal{F}, \mathbb{P})$ with a filtration $(\mathcal{F}(t))_{t \geq 0}$ satisfying the usual conditions, and let $(B(t): t \geq 0)$ be a standard $m$-dimensional Brownian motion on this space. We study the following SDE with time delay:

$$
\begin{aligned}
d X(t) & =L\left(X_{t}\right) d t+\Sigma d B(t) \quad \text { for } t \geq 0, \\
X(t) & =\phi(t) \quad \text { for } t \in[-\tau, 0],
\end{aligned}
$$

where $L$ is a continuous and linear functional on $C\left([-\tau, 0] ; \mathbb{R}^{d}\right)$ for a constant $\tau \geq 0$, and $\Sigma$ is a $d \times m$ matrix with real entries.

For every $\phi \in C\left([-\tau, 0] ; \mathbb{R}^{d}\right)$ there exists a unique, adapted strong solution $(X(t, \phi): t \geq-\tau)$ with finite second moments of (2.7) (cf., e.g., Mao [39]). The dependence of the solutions on the initial condition $\phi$ is neglected in our notation in what follows; that is, we will write $x(t)=x(t, \phi)$ and $X(t)=X(t, \phi)$ for the solutions of (2.2) and (2.7), respectively.

By Reiss, Riedle, and van Gaans [54, Lemma 6.1] the solution $(X(t): t \geq-\tau)$ of (2.7) obeys a variation-of-constants formula

$$
X(t)=\left\{\begin{array}{cc}
x(t)+\int_{0}^{t} r(t-s) \Sigma d B(s), & t \geq 0, \\
\phi(t), & t \in[-\tau, 0],
\end{array}\right.
$$

where $r$ is the fundamental solution of (2.2). The process $X$ defined by (2.8) obeys (2.7) pathwise on an almost sure event.

In this paper, we let $\langle\cdot, \cdot\rangle$ stand for the standard inner product on $\mathbb{R}^{d}$ and $|\cdot|_{2}$ for the standard Euclidean norm induced from it. We also let $|\cdot|_{\infty}$ stand for the infinity norm on $\mathbb{R}^{d}$, and if $\phi \in C\left([-\tau, 0] ; \mathbb{R}^{d}\right)$, we define $\|\phi\|_{2}=\sup _{-\tau \leq s \leq 0}|\phi(s)|_{2}$. By way of clarification, we note that here $|\cdot|_{\infty}$ stands for a vector norm rather than a norm on a space of continuous functions. For $i=1, \ldots, d$, the $i$ th standard basis vector in $\mathbb{R}^{d}$ is denoted $\mathbf{e}_{i}$. If $X$ and $Y$ are two random variables, then we denote the correlation and the covariance between $X$ and $Y$ by $\operatorname{Corr}(X, Y)$ and $\operatorname{Cov}(X, Y)$, respectively.

3. Statement and discussion of main results. In the first part of this section we state and discuss the main results of the paper. The second subsection is devoted to a discussion of the limitations of techniques used in this paper and attempts to give a programme for extending the results to other classes of affine SFDEs to which the technical assumptions employed here would not apply.

3.1. Main results. We start with some preparatory lemmata used to establish the almost sure rate of growth of the running maxima of the solution of a scalar version of (2.7).

Lemma 2. Suppose $\left(a_{n}\right)_{n=1}^{\infty}$ is a real sequence with $\lim _{\sup _{n \rightarrow \infty}} a_{n} \geq 0$ and $\gamma$ is a nonnegative and nondecreasing sequence, with $\gamma(n) \rightarrow \infty$ as $n \rightarrow \infty$. Then

$$
\limsup _{n \rightarrow \infty} \frac{\max _{1 \leq j \leq n} a_{j}}{\gamma(n)}=\limsup _{n \rightarrow \infty} \frac{a_{n}}{\gamma(n)} .
$$

The above lemma is a slight generalization and analogue of Lemma 2.6.3 in [39], which is stated at the end of this section. A proof of this result is postponed to the final section. 
The next result gives precise information on the growth of the running maxima of a sequence of normal random variables which have an exponentially decaying autocovariance function.

Lemma 3. Suppose $\left(X_{n}\right)_{n=1}^{\infty}$ is a sequence of jointly normal standard random variables satisfying

$$
\left|\operatorname{Cov}\left(X_{i}, X_{j}\right)\right| \leq \lambda^{|i-j|}
$$

for some $\lambda \in(0,1)$. Then

$$
\lim _{n \rightarrow \infty} \frac{\max _{1 \leq j \leq n} X_{j}}{\sqrt{2 \log n}}=1 \quad \text { a.s. }
$$

These lemmata are used to determine the size of the large fluctuations of the solution of (2.7) in the scalar case, i.e., the case in which $d=1$ and the solution $X$ of (2.7) is a one-dimensional process. If $m>1$ and $\Sigma=\left(\Sigma_{1}, \Sigma_{2}, \ldots, \Sigma_{m}\right)$ is a $1 \times m$ matrix, we note that the martingale

$$
M(t)=\sum_{j=1}^{m} \int_{0}^{t} \Sigma_{j} d B_{j}(s), \quad t \geq 0,
$$

can be rewritten as

$$
M(t)=\int_{0}^{t} \sigma d W(s), \quad t \geq 0,
$$

where $\sigma=\left(\sum_{j=1}^{m} \Sigma_{j}^{2}\right)^{1 / 2}$ and $W$ is a one-dimensional Brownian motion. Therefore, in the scalar case it suffices to study the equation

$$
\begin{aligned}
d X(t) & =L\left(X_{t}\right) d t+\sigma d W(t) \quad \text { for } t \geq 0, \\
X(t) & =\phi(t) \quad \text { for } t \in[-\tau, 0],
\end{aligned}
$$

where $\phi \in C([-\tau, 0] ; \mathbb{R})$.

THEOREM 1. Suppose that $r$ is the solution of $(2.3)$ with $d=1$ and that $v_{0}(\nu)<0$, where $v_{0}(\nu)$ is defined as (2.5). Let $X$ be the unique continuous adapted process which obeys (3.2). Then

$$
\limsup _{t \rightarrow \infty} \frac{|X(t)|}{\sqrt{2 \log t}}=|\sigma| \sqrt{\int_{0}^{\infty} r^{2}(s) d s}=: \Gamma \quad \text { a.s. }
$$

Moreover,

$$
\begin{aligned}
& \limsup _{t \rightarrow \infty} \frac{X(t)}{\sqrt{2 \log t}}=|\sigma| \sqrt{\int_{0}^{\infty} r^{2}(s) d s} \quad \text { a.s. } \\
& \liminf _{t \rightarrow \infty} \frac{X(t)}{\sqrt{2 \log t}}=-|\sigma| \sqrt{\int_{0}^{\infty} r^{2}(s) d s} \quad \text { a.s. }
\end{aligned}
$$

Theorem 1 can be applied in the case where $X$ is a mean-reverting OrnsteinUhlenbeck (OU) process. Consider the OU process governed by the following equation:

$$
d U(t)=-\alpha U(t) d t+\sigma d B(t), \quad t \geq 0
$$

Copyright $@$ by SIAM. Unauthorized reproduction of this article is prohibited. 
with $U(0)=u_{0}$ and $\alpha>0$. Then $U$ is a Gaussian process and has a limiting distribution $N\left(0, \sigma^{2} / 2 \alpha\right)$. It can easily be shown that $e^{\alpha t} U(t)=u_{0}+M(t)$, where $M(t)=\sigma \int_{0}^{t} e^{\alpha s} d B(s)$ is a continuous martingale with quadratic variation $\gamma(t):=$ $\sigma^{2}\left(\left(e^{2 \alpha t}-1\right) / 2 \alpha\right.$. By the time-change theorem for martingales [32, Theorem 3.4.6], $M\left(\gamma^{-1}(t)\right)$ is a standard Brownian motion. Hence by the law of the iterated logarithm for standard one-dimensional Brownian motion,

$$
\limsup _{t \rightarrow \infty} \frac{\left|M\left(\gamma^{-1}(t)\right)\right|}{\sqrt{2 t \log \log t}}=1 \quad \text { a.s. }
$$

which implies

$$
\limsup _{t \rightarrow \infty} \frac{|U(t)|}{\sqrt{2 \log t}}=\frac{|\sigma|}{\sqrt{2 \alpha}} \text { a.s. }
$$

Thus it can be seen in this simple case that a short and independent proof of (3.3) can be given. In the general case with linear distributed delay, the solution of (3.2) can be represented by (2.8). Moreover, under the condition $v_{0}(\nu)<0$, the solution is asymptotically Gaussian distributed with mean zero and variance $\Gamma^{2}$. However, since the characteristic equation of $r$ in general has infinitely many roots, it is difficult to write an explicit solution for $r$ and hence for $X$. Consequently, the value of $\Gamma$ is not easy to compute. Moreover, since the process given by the stochastic integral in (2.8) is not in general a martingale, the martingale time-change approach given above for the OU process is not available. We therefore use Mill's estimate together with Lemma 3 (both on Gaussian random variables) to prove (3.3) on a sequence of mesh points $a_{n}$. Then we investigate the behavior of the solution in continuous time by choosing $a_{n}$ so that the distance between the mesh points tends to zero as $n \rightarrow \infty$. This enables us to closely control the behavior of $X$ on the interval $\left[a_{n}, a_{n+1}\right]$.

The condition $v_{0}(\nu)<0$ is essential in Theorem 1. Appleby, Riedle, and Swords (cf. [6]) studied the case when $v_{0}(\nu) \geq 0$. Under some additional conditions on $v_{0}(\nu)$ which assume that the zero of the characteristic equation with the largest real part is simple and real, their results can be summarized as follows:

(a) If $v_{0}(\nu)=0$, then

$$
\limsup _{t \rightarrow \infty} \frac{|X(t)|}{\sqrt{2 t \log \log t}}=L_{1} \quad \text { a.s. }
$$

(b) If $v_{0}(\nu)>0$, then

$$
\lim _{t \rightarrow \infty} e^{-v_{0}(\nu) t} X(t)=L_{2} \quad \text { a.s. }
$$

where $L_{1}$ is deterministic and $L_{2}$ is a random variable. Theorem 1 , together with these two results, connects the location of the roots of the characteristic equation to the asymptotic behavior of the resolvent $r$ and hence to the asymptotic behavior of the stochastic process $X$. If the underlying deterministic equation is stable in such a way that the resolvent tends to zero $\left(v_{0}(\nu)<0\right)$, then the process is asymptotically stationary and Gaussian. If mean-reverting forces in the system are just compensated by positive feedback away from the underlying deterministic equilibrium $\left(v_{0}(\nu)=0\right)$, then the process obeys the law of the iterated logarithm and is nonstationary (but has increments which are asymptotically stationary), and therefore has properties similar to a standard Brownian motion, while at the same time it possesses dependent 
increments. Finally, if the resolvent is exponentially unstable $\left(v_{0}(\nu)>0\right)$, then the process is exponentially transient.

An interesting and special case to which Theorem 1 can be applied arises when $X$ is governed by the generalized Langevin equation

$$
d X(t)=[a X(t)+b X(t-\tau)] d t+\sigma d B(t), \quad t \geq 0,
$$

where $a, b \in \mathbb{R}$ and $\tau>0$. Küchler and Mensch [34] studied this equation in great detail. One important contribution of their work is that the conditions on $a, b$, and $\tau$ which ensure the stationarity of the solution are classified. An explicit solution of the resolvent $r$ in terms of elementary functions can be found by the method of steps, which is also given in [34]. Therefore the constant $\Gamma$ for the solution of (3.6) can be approximated to an arbitrary precision by an explicit formula with finitely many terms. Naturally, for a general $r$, we can use deterministic numerical methods to approximate $\Gamma$ to any desired precision, but such methods will not yield a formula for the approximation.

We can extend the result of Theorem 1 to the solution of the general finitedimensional equation (2.7).

THEOREM 2. Suppose that $r$ is the solution of $(2.3)$ and that $v_{0}(\nu)<0$, where $v_{0}(\nu)$ is defined as (2.5). Let $X$ be the unique continuous adapted d-dimensional process which obeys (2.7). Then, for each $1 \leq i \leq d$,

$$
\limsup _{t \rightarrow \infty} \frac{X_{i}(t)}{\sqrt{2 \log t}}=\sigma_{i} \quad \text { and } \quad \liminf _{t \rightarrow \infty} \frac{X_{i}(t)}{\sqrt{2 \log t}}=-\sigma_{i} \quad \text { a.s. },
$$

where

$$
\sigma_{i}=\sqrt{\sum_{k=1}^{m} \int_{0}^{\infty} \rho_{i k}^{2}(s) d s}
$$

and $\rho(t)=r(t) \Sigma \in \mathbb{R}^{d \times m}$. Moreover,

$$
\limsup _{t \rightarrow \infty} \frac{|X(t)|_{\infty}}{\sqrt{2 \log t}}=\max _{i=1, \ldots, d} \sigma_{i} \quad \text { a.s. }
$$

Our final main result shows that (2.7) can be perturbed by a nonlinear functional $N$ in the drift (which is of lower than linear order at infinity) without changing the asymptotic behavior of the underlying affine SFDE. To make this claim more precise, we characterize the perturbing nonlinear functional $N$ as follows. Suppose $N:[0, \infty) \times C[-\tau, 0] \rightarrow \mathbb{R}^{d}$ obeys the following:

$$
\begin{aligned}
& \text { For all } n \in \mathbb{N} \text { there exists a } K_{n}>0 \text { such that if } \varphi, \psi \in C\left([-\tau, 0] ; \mathbb{R}^{d}\right) \\
& \text { obey }\|\varphi\|_{2} \vee\|\psi\|_{2} \leq n \text {, then }|N(t, \varphi)-N(t, \psi)|_{2} \leq K_{n}\|\varphi-\psi\|_{2}, \\
& \text { and } N \text { is continuous in its first argument; }
\end{aligned}
$$

and

$$
\lim _{\|\varphi\|_{2} \rightarrow \infty} \frac{|N(t, \varphi)|_{2}}{\|\varphi\|_{2}}=0 \quad \text { uniformly in } t
$$

and

$$
t \mapsto|N(t, 0)|_{2} \text { is bounded on }[0, \infty)
$$


Before stating our main result, we examine the hypotheses (3.10)-(3.12) and prove an important estimate deriving therefrom. By the hypothesis (3.11), we mean that for every $\varepsilon>0$ there is a $\Phi=\Phi(\varepsilon)>0$ such that if $\varphi \in C\left([-\tau, 0] ; \mathbb{R}^{d}\right)$ obeys $\|\varphi\|_{2} \geq \Phi(\varepsilon)$, we then have

$$
|N(t, \varphi)|_{2} \leq \varepsilon\|\varphi\|_{2} \text { for all } t \geq 0
$$

By (3.12), we have that there is an $\bar{n} \geq 0$ such that $|N(t, 0)|_{2} \leq \bar{n}$ for all $t \geq 0$. Also by (3.10), for all $\varphi$ such that $\|\varphi\|_{2} \leq\lceil\Phi(\varepsilon)\rceil$ (where $\lceil x\rceil$ denotes the smallest integer greater than or equal to $x \geq 0$ ) we have that there is a $K(\varepsilon)=K_{\lceil\Phi(\varepsilon)\rceil}$ such that

$$
|N(t, \varphi)|_{2} \leq|N(t, \varphi)-N(t, 0)|_{2}+|N(t, 0)|_{2} \leq K(\varepsilon)\|\varphi\|_{2}+\bar{n} \leq K(\varepsilon)\lceil\Phi(\varepsilon)\rceil+\bar{n} .
$$

Therefore with $L(\varepsilon):=K(\varepsilon)\lceil\Phi(\varepsilon)\rceil+\bar{n}$ we have

$$
|N(t, \varphi)|_{2} \leq L(\varepsilon) \text { for all } t \geq 0 \text { and all }\|\varphi\|_{2} \leq \Phi(\varepsilon) .
$$

Hence for every $\varepsilon>0$ there exists $L(\varepsilon)>0$ such that

$$
|N(t, \varphi)|_{2} \leq L(\varepsilon)+\varepsilon\|\varphi\|_{2} \quad \text { for all } t \geq 0 \text { and all } \varphi \in C\left([-\tau, 0] ; \mathbb{R}^{d}\right) .
$$

The hypothesis (3.12) ensures that the functional $N$ is (in some sense) close to being an autonomous functional or is bounded by an autonomous functional.

We study the following nonlinear SDE with time delay:

$$
\begin{aligned}
d X(t) & =\left(L\left(X_{t}\right)+N\left(t, X_{t}\right)\right) d t+\Sigma d B(t) \quad \text { for } t \geq 0, \\
X(t) & =\phi(t) \quad \text { for } t \in[-\tau, 0],
\end{aligned}
$$

where $L$ is a continuous and linear functional on $C\left([-\tau, 0] ; \mathbb{R}^{d}\right)$ for a constant $\tau \geq 0$, and $\Sigma$ is a $d \times m$ matrix with real entries.

Since $L$ is linear and $N$ obeys (3.10) and (3.11), for every $\phi \in C\left([-\tau, 0] ; \mathbb{R}^{d}\right)$ there exists a unique, adapted strong solution $(X(t, \phi): t \geq-\tau)$ with finite second moments of (3.14) (cf., e.g., Mao [39]).

Theorem 3. Suppose that $N$ obeys (3.10), (3.11), and (3.12). Also suppose that $r$ is the solution of $(2.3)$ and $v_{0}(\nu)<0$, where $v_{0}(\nu)$ is defined as (2.5). Let $X$ be the unique continuous adapted d-dimensional process which obeys (3.14). Then, for each $1 \leq i \leq d$,

$$
\limsup _{t \rightarrow \infty} \frac{X_{i}(t)}{\sqrt{2 \log t}}=\sigma_{i} \quad \text { and } \quad \liminf _{t \rightarrow \infty} \frac{X_{i}(t)}{\sqrt{2 \log t}}=-\sigma_{i} \quad \text { a.s. }
$$

where $\sigma_{i}$ is given by (3.8). Moreover,

$$
\limsup _{t \rightarrow \infty} \frac{|X(t)|_{\infty}}{\sqrt{2 \log t}}=\max _{1 \leq i \leq d} \sigma_{i} \quad \text { a.s. }
$$

Since, in general, it is not possible to obtain a representation that is analogous to (2.8) for nonlinear equations such as (3.14), the proof cannot directly rely on Gaussianity of the process. Instead, by using a comparison argument, we conclude that if the nonlinear term in the drift is smaller than linear order at infinity (cf. assumption (3.11)), the size of the large fluctuations of a Gaussian stationary process 
is retained. Due to the presence of the supremum norm estimates for $N$, the proof involves the construction of Halanay-type functional differential inequalities. This technique is frequently used in [8].

The following auxiliary lemma is required in the proof of Theorem 3; its proof is deferred to the final section.

Lemma 4. Let $\vartheta$ be positive and nondecreasing with $\vartheta(t-T) / \vartheta(t) \rightarrow 1$, as $t \rightarrow \infty$, for all $T \geq 0$. If $\kappa$ is nonnegative with $\int_{0}^{\infty} \kappa(s) d s \in(0, \infty)$, then

$$
\lim _{t \rightarrow \infty} \frac{1}{\vartheta(t)} \int_{0}^{t} \kappa(t-s) \vartheta(s) d s=\int_{0}^{\infty} \kappa(s) d s .
$$

We also need the following continuous analogue of Lemma 2, which appeared as Lemma 2.6.3 in [39].

Lemma 5. Suppose $y:[0, \infty) \rightarrow[0, \infty)$, and let $\vartheta:[0, \infty) \rightarrow(0, \infty)$ be a nondecreasing function with $\vartheta(t) \rightarrow \infty$ as $t \rightarrow \infty$. Then

$$
\limsup _{t \rightarrow \infty} \frac{\max _{0 \leq s \leq t} y(s)}{\vartheta(t)}=\limsup _{t \rightarrow \infty} \frac{y(t)}{\vartheta(t)} .
$$

3.2. Alternative approaches and generalization. Although we have succeeded in establishing satisfactory results on the large fluctuations of solutions of (2.7), our results hinge on two key properties of the differential resolvent $r$ satisfying (2.3). The first is that $r$ is in $C^{1}\left((0, \infty) ; \mathbb{R}^{d}\right)$; the second is that $r$ decays exponentially fast because $v_{0}(\nu)<0$. However, it is easy to formulate affine SFDEs for which neither of these properties holds.

For instance, consider the scalar Volterra equation

$$
d X(t)=\int_{[0, t]} \nu(d s) X(t-s) d t+\Sigma d B(t), \quad t \geq 0,
$$

where $\nu \in M([0, \infty) ; \mathbb{R})$. Suppose now that $\nu(d s)=a \delta_{0}(d s)+k(s) d s$, where $k$ is a positive, continuous, and integrable function, $\delta_{0}$ is the Dirac measure at 0 , and $a+\int_{0}^{\infty} k(s) d s<0$. In this case, $X$ still obeys the variation-of-constants formula

$$
X(t)=r(t) X(0)+\int_{0}^{t} r(t-s) \Sigma d B(s), \quad t \geq 0,
$$

where in this case the differential resolvent $r$ obeys

$$
r^{\prime}(t)=\int_{[0, t]} \nu(d s) r(t-s), \quad t \geq 0 ; \quad r(0)=1 .
$$

The conditions on $a$ and $k$ guarantee that $r \in L^{1}(0, \infty)$ (see [16]). However, if $k$ decays subexponentially in a sense defined in, e.g., [3] (which implies that $k(t) e^{\varepsilon t} \rightarrow \infty$ as $t \rightarrow \infty$ for each $\varepsilon>0$ ), then it can be shown that $t \mapsto r(t) / k(t)$ tends to a nontrivial limit as $t \rightarrow \infty$ (see, e.g., [3]). Therefore $r$ cannot decay exponentially, and so we cannot use Lemma 3 to obtain a lower bound.

On the other hand, if we consider the scalar neutral affine SFDE

$$
d\left(X(t)-\int_{[-\tau, 0]} \mu(d s) X(t+s)\right)=\int_{[-\tau, 0]} \nu(d s) X(t+s) d t+\Sigma d B(t), \quad t \geq 0
$$


then $X$ obeys the variation-of-constants formula

$$
X(t)=x(t)+\int_{0}^{t} \rho(t-s) \Sigma d B(s), \quad t \geq 0,
$$

where $x$ is the solution of the underlying deterministic linear neutral differential equation with the same initial condition as $X$, and $\rho$ is the resolvent given by

$$
\begin{aligned}
\frac{d}{d t}\left(\rho(t)-\int_{[-\tau, 0]} \mu(d s) \rho(t+s)\right) & =\int_{[-\tau, 0]} \nu(d s) \rho(t+s), \quad t \geq 0 \\
\rho(0) & =1 ; \quad \rho(t)=0, \quad t \in[-\tau, 0) .
\end{aligned}
$$

If $\rho$ is integrable, then we are guaranteed that $\rho$ will decay to zero exponentially fast (cf. e.g., [29]). However, we are not guaranteed that $\rho$ will be differentiable, even though $\rho$ is continuous (cf., e.g., [29]). Therefore, the proof of the upper bound on $|X|$ in Theorem 1 cannot be continued in the same manner as outlined in section 4 .

By examining these two examples, it seems to be advantageous to develop general methods to determine the asymptotic behavior of the process

$$
\tilde{X}(t)=\int_{0}^{t} r(t-s) d B(s), \quad t \geq 0,
$$

where $r \in L^{2}\left([0, \infty) ; \mathbb{R}^{d \times d}\right)$ is continuous, in order to determine asymptotic properties for general affine SFDEs, including the important classes of Volterra and neutral equations. We identify several strategies which are worthy of investigation, and some ideas as to their possible implementation are given here. One might also hope that these methods could unify (and perhaps simplify) the proofs for all the above-mentioned classes of affine equations. To make our discussion simple, we focus on the scalar case $(d=1)$.

The first alternative strategy derives from the fact that the process $\tilde{X}$ is nearly stationary. The main idea is to consider the asymptotic behavior of $\tilde{X}$ by writing

$$
\tilde{X}(t)=\int_{-\infty}^{t} r(t-s) d B(s)-\int_{-\infty}^{0} r(t-s) d B(s)=: X_{1}(t)+X_{2}(t)
$$

where the standard Brownian motion $B$ has been extended independently to $(-\infty, 0)$.

Before proceeding further, we interpret and justify the existence of the infinite integrals in (3.18). Since $r \in L^{2}(0, \infty)$ and $r$ is continuous, for each fixed $t \geq 0$, the infinite integral

$$
-X_{2}(t)=\int_{-\infty}^{0} r(t-s) d B(s)
$$

can be defined as the almost sure or mean square limit as $T \rightarrow \infty$ of the well-defined Gaussian random variables

$$
\int_{-T}^{0} r(t-s) d B(s)
$$

with the limit $X_{2}$ itself being Gaussian. If we think of $X_{2}$ as a process, we view $X_{2}(t)$ for all $t \geq 0$ as random variables measurable with respect to the common $\sigma$-algebra 
$\mathcal{F}^{B}(0)$. Once this has been defined, we can define $X_{1}(t)$ according to

$$
X_{1}(t)=\int_{-\infty}^{t} r(t-s) d B(s):=\int_{0}^{t} r(t-s) d B(s)+\int_{-\infty}^{0} r(t-s) d B(s) .
$$

Since $\tilde{X}$ is adapted to $\mathcal{F}^{B}$, it follows that $X_{1}$ is also adapted to $\mathcal{F}^{B}$.

The first term on the right-hand side of (3.18) is a stationary Gaussian process with autocovariance function $c$ given by

$$
c(h)=\int_{0}^{\infty} r(u) r(u+h) d u, \quad h \geq 0 .
$$

By a result of Marcus [44] we can show that $X_{1}$ obeys

$$
\limsup _{t \rightarrow \infty} \frac{\left|X_{1}(t)\right|}{\sqrt{2 \log t}} \leq \sqrt{\int_{0}^{\infty} r^{2}(s) d s} \text { a.s. }
$$

On the other hand, by [51, Theorem 5.2] we have that $c(h) \log h \rightarrow 0$ as $h \rightarrow \infty$ implies

$$
\liminf _{t \rightarrow \infty}\left\{\sup _{0 \leq s \leq t} X_{1}(s)-\sqrt{\int_{0}^{\infty} r^{2}(s) d s} \sqrt{2 \log t}\right\} \geq 0 \quad \text { a.s. }
$$

Combining these results gives

$$
\limsup _{t \rightarrow \infty} \frac{X_{1}(t)}{\sqrt{2 \log t}}=\limsup _{t \rightarrow \infty} \frac{\left|X_{1}(t)\right|}{\sqrt{2 \log t}}=\sqrt{\int_{0}^{\infty} r^{2}(s) d s} \quad \text { a.s. }
$$

Then Theorem 1 follows provided that we can prove that

$$
\lim _{t \rightarrow \infty} \frac{X_{2}(t)}{\sqrt{2 \log t}}=0 \quad \text { a.s. }
$$

The proof of the claim (3.19) is made plausible by the fact that (a) $X_{2}$ is a Gaussian process and that (b) $\mathbb{E}\left[X_{2}^{2}(t)\right]=\int_{t}^{\infty} r^{2}(u) d u \rightarrow 0$ as $t \rightarrow \infty$. The latter observation seems to suggest that $X_{2}$ should be asymptotically dominated by $X_{1}$, which is a process with constant rather than asymptotically vanishing variance. This approach seems to hold out the prospect that the assumption that $r$ (and therefore the autocovariance function $c$ ) decays exponentially can be significantly relaxed.

Another related approach is to use results of Deo [24, 23] on nonstationary Gaussian sequences of random variables. This avoids a proof of an asymptotic estimate such as (3.19) for the residual process $X_{2}$. Since it transpires that the upper bound on $\tilde{X}$ can be established without the need for exponential estimates on $r$, it remains to establish that this upper bound is sharp by determining a related lower bound on the growth of the large fluctuations. In order to prove this, one might consider a sequence of Gaussian random variables sampled from the continuous-time process $\tilde{X}$, and this is what motivates our proposal to use Deo's results. He shows that if there is a sequence of $N(0,1)$ random variables $\left(X_{n}\right)_{n \geq 1}$ for which $c(i, j)=\mathbb{E}\left[X_{i} X_{j}\right]$ obeys

$$
\sum_{n=1}^{\infty} \delta_{n}^{2}<+\infty, \quad \text { where } \quad \delta_{n}:=\sup _{|i-j| \geq n}|c(i, j)|,
$$

Copyright $@$ by SIAM. Unauthorized reproduction of this article is prohibited. 
then

$$
\lim _{n \rightarrow \infty} \max _{1 \leq j \leq n}\left\{X_{j}-\sqrt{2 \log n}\right\}=0 \quad \text { a.s. }
$$

The essential thrust of our proof is then as follows: first, we construct a sequence of normal random variables $X_{n}:=\tilde{X}(n) / \sqrt{v(n)}$, where $\tilde{X}$ is given by (3.17). By virtue of the fact that $r \in L^{2}(0, \infty)$, we then would hope to show that the sequence $\left(X_{n}\right)_{n \geq 1}$ obeys (3.20). This would enable us to prove our results without a stipulation on the exponential decay of $r$. Our conjecture is given some weight by the fact that $r \in L^{1}(0, \infty)$ forces the upper estimate on the autocovariance function $\bar{c}$ of $\tilde{X}$ given by

$$
\bar{c}(h)=\int_{0}^{\infty}|r(u)||r(u+h)| d u
$$

to obey $\bar{c} \in L^{2}(0, \infty)$. This fact is in some sense analogous to the discrete square summability condition (3.20), and the aim of our proof is then to demonstrate that $c \in L^{2}(0, \infty)$ implies $(3.20)$, perhaps when taken in conjunction with some harmless technical conditions on $r$.

Yet another potential approach is to consider the process $\tilde{X}$ defined in (3.17) as the increment process of a nonstationary Gaussian process. In recent years an impressive literature has developed on the asymptotic and path behavior of increments of Gaussian processes, in particular of fractional Brownian motion. To give a full picture of this research would be difficult, but some representative papers relevant to this discussion include $[18,19,20,21,49,56,57]$. A tentative manner in which this theory might be applied is as follows. Suppose (as in the case of fractional Brownian motion) that a nonstationary Gaussian process can be represented in the integral form

$$
X(t)=\int_{0}^{t}(c+\rho(t-s)) d B(s), \quad t \geq 0,
$$

where $c>0$, and suppose that $\rho$ is in $L^{2}(0, \infty)$ and obeys $\rho(t)=0$ for all $t<0$. Let $\delta>0$. Then the so-called lag increment $X(t)-X(t-\delta)$ is given by

$$
\int_{0}^{t} \rho_{\delta}(t-s) d B(s), \quad t \geq \delta,
$$

where $\rho_{\delta}(t)=\rho(t)-\rho(t-\delta)$ is in $L^{2}(0, \infty)$. Hence the lag increment is of a form similar to the classes of processes considered in this paper. Therefore a study of the large fluctuations of the lag increments of the nonstationary Gaussian process $X$ is strongly related to determining the large deviations of the processes considered in this work. However, the question remains open as to how one might choose the lag $\delta$ in an appropriate manner in order to apply this theory to the processes studied here.

Finally, it should be remarked that solutions of affine SDDEs can be thought of as solutions of a Cauchy problem in a Hilbert space (see, e.g., Da Prato and Zabczyk [22]). One of the advantages of this approach is that the solutions in this abstract setting are Markovian, whereas solutions in the original finite-dimensional space are in general non-Markovian. This method of studying SDDEs has led to excellent results on qualitative features on the existence and uniqueness of stationary solutions of such equations (see, e.g., Riedle and van Neerven [55], Reiss, Riedle, and van Gaans [53]), but to the best of the authors' knowledge it has not yet been applied to obtain quantitative results of the type demanded in the current work. Moreover, 
since solutions exhibit correlation over time whether they are Markov or not, it is not immediately clear that the autocorrelation of solutions, which is at the heart of the difficulties involved in determining large fluctuations, would be appreciably simplified in this setting. However, such an abstract approach should be of great value for determining the large deviation properties of solutions of retarded affine stochastic partial differential equations (see, e.g., $[36,37]$ ), which would not yield readily to the real-space techniques developed in this paper.

\section{Proofs of theorems.}

4.1. Proof of Theorem 1. Since $v_{0}(\nu)<0$, we have that $r(t) \rightarrow 0$ as $t \rightarrow \infty$, so the first term on the right-hand side of (2.8) tends to zero as $t \rightarrow \infty$. We analyze the behavior of the second term. We first establish

$$
\limsup _{t \rightarrow \infty} \frac{|X(t)|}{\sqrt{2 \log t}} \leq|\sigma| \sqrt{\int_{0}^{\infty} r^{2}(s) d s} \quad \text { a.s. }
$$

Define

$$
\tilde{X}(t):=\sigma \int_{0}^{t} r(t-s) d B(s)
$$

It is helpful to define

$$
v(t)=\sigma^{2} \int_{0}^{t} r^{2}(s) d s, \quad t \geq 0
$$

Then $\tilde{X}(t)$ is normally distributed with mean 0 and variance $v(t)$, where $v$ is given by (4.2). Since $r \in L^{1}([0, \infty) ; \mathbb{R})$, by Lemma 1 we have $r(t) \rightarrow 0$ as $t \rightarrow \infty$. By continuity, $r$ is bounded, and so $r \in L^{2}([0, \infty) ; \mathbb{R})$. Therefore

$$
v(t)=\sigma^{2} \int_{0}^{t} r^{2}(s) d s \leq \sigma^{2} \int_{0}^{\infty} r^{2}(s) d s=: \Gamma^{2} .
$$

Let $\theta \in(0,1)$. Clearly $\lim _{t \rightarrow \infty} v(t)=\Gamma^{2}$ and $\lim _{n \rightarrow \infty} v\left(n^{\theta}\right)=\Gamma^{2}$. If $Z\left(n^{\theta}\right):=$ $\tilde{X}\left(n^{\theta}\right) / \sqrt{v\left(n^{\theta}\right)}$, by using a proof similar to that in Lemma 8 in section 5 , we obtain

$$
\limsup _{n \rightarrow \infty} \frac{\left|Z\left(n^{\theta}\right)\right|}{\sqrt{2 \log n}} \leq 1 \quad \text { a.s. }
$$

Therefore

$$
\limsup _{n \rightarrow \infty} \frac{\left|\tilde{X}\left(n^{\theta}\right)\right|}{\sqrt{2 \log n}} \leq \Gamma \quad \text { a.s. }
$$

Now, by a stochastic Fubini theorem (cf., e.g., [52, Chapter IV.6, Theorem 64]), we get

$$
\begin{aligned}
\tilde{X}(t) & =\sigma \int_{0}^{t}\left(1+\int_{0}^{t-s} r^{\prime}(u) d u\right) d B(s) \\
& =\sigma B(t)+\sigma \int_{0}^{t} \int_{s}^{t} r^{\prime}(u-s) d u d B(s) \\
& =\sigma B(t)+\sigma \int_{0}^{t} \int_{0}^{u} r^{\prime}(u-s) d B(s) d u .
\end{aligned}
$$

Copyright (c) by SIAM. Unauthorized reproduction of this article is prohibited. 
Therefore

$$
|\tilde{X}(t)| \leq \sigma\left|B(t)-B\left(n^{\theta}\right)\right|+\sigma\left|\int_{n^{\theta}}^{t} \int_{0}^{u} r^{\prime}(u-s) d B(s) d u\right|+\left|\tilde{X}\left(n^{\theta}\right)\right| .
$$

We now consider each of the three terms on the left-hand side of (4.5). By the properties of a standard Brownian motion, we have

$$
\begin{aligned}
\mathbb{P}\left[\sup _{n^{\theta} \leq t \leq(n+1)^{\theta}}\left|B(t)-B\left(n^{\theta}\right)\right|>1\right] & \leq 2 \mathbb{P}\left[\sup _{0 \leq t \leq(n+1)^{\theta}-n^{\theta}} B(t)>1\right] \\
& =2 \mathbb{P}\left[\left|B\left((n+1)^{\theta}-n^{\theta}\right)\right|>1\right] \\
& =4 \mathbb{P}\left[Z>\frac{1}{\sqrt{(n+1)^{\theta}-n^{\theta}}}\right]
\end{aligned}
$$

where $Z$ is a standard normal random variable. Since $\left\{(n+1)^{\theta}-n^{\theta}\right\} / n^{\theta-1} \rightarrow \theta$ as $n \rightarrow \infty$, by Mill's estimate and the Borel-Cantelli lemma, there exists $N(\omega) \in \mathbb{N}$ such that for all $n>N$

$$
\sup _{n^{\theta} \leq t \leq(n+1)^{\theta}}\left|B(t)-B\left(n^{\theta}\right)\right| \leq 1 \quad \text { a.s. }
$$

That is,

$$
\limsup _{n \rightarrow \infty} \sup _{n^{\theta} \leq t \leq(n+1)^{\theta}}\left|B(t)-B\left(n^{\theta}\right)\right| \leq 1 \quad \text { a.s. }
$$

For the double integral term in (4.5), define

$$
U_{n}:=\sup _{n^{\theta} \leq t \leq(n+1)^{\theta}}\left|\int_{n^{\theta}}^{t} \int_{0}^{u} r^{\prime}(u-s) d B(s) d u\right| .
$$

Then, by Hölder's inequality

$$
\begin{aligned}
\mathbb{E}\left[U_{n}^{2 k}\right] & \leq \mathbb{E}\left[\sup _{n^{\theta} \leq t \leq(n+1)^{\theta}}\left(\int_{n^{\theta}}^{t}\left|\int_{0}^{u} r^{\prime}(u-s) d B(s)\right| d u\right)^{2 k}\right] \\
& \leq \mathbb{E}\left[\sup _{n^{\theta} \leq t \leq(n+1)^{\theta}}\left(t-n^{\theta}\right)^{2 k-1} \int_{n^{\theta}}^{t}\left|\int_{0}^{u} r^{\prime}(u-s) d B(s)\right|^{2 k} d u\right] \\
& =\mathbb{E}\left[\left((n+1)^{\theta}-n^{\theta}\right)^{2 k-1} \int_{n^{\theta}}^{(n+1)^{\theta}}\left|\int_{0}^{u} r^{\prime}(u-s) d B(s)\right|^{2 k} d u\right] \\
& =\left((n+1)^{\theta}-n^{\theta}\right)^{2 k-1} \int_{n^{\theta}}^{(n+1)^{\theta}} \mathbb{E}\left|\int_{0}^{u} r^{\prime}(u-s) d B(s)\right|^{2 k} d u .
\end{aligned}
$$

Now, for $u \geq 0, \int_{0}^{u} r^{\prime}(u-s) d B(s)$ is a Gaussian process with mean 0 and variance $\int_{0}^{u} r^{\prime}(s)^{2} d s$. Since $r$ decays exponentially by Lemma 1, the variance is bounded above by $\int_{0}^{\infty} r^{\prime}(s)^{2} d s=: L$. Hence there exists $C_{k}>0$ such that

$$
\int_{n^{\theta}}^{(n+1)^{\theta}} \mathbb{E}\left|\int_{0}^{u} r^{\prime}(u-s) d B(s)\right|^{2 k} d u \leq \int_{n^{\theta}}^{(n+1)^{\theta}} C_{k} L^{k} d u=C_{k} L^{k}\left((n+1)^{\theta}-n^{\theta}\right) .
$$

Copyright (c) by SIAM. Unauthorized reproduction of this article is prohibited. 
By Chebyshev's inequality, we therefore get

$$
\mathbb{P}\left(\left|U_{n}\right| \geq 1\right) \leq \mathbb{E}\left[U_{n}^{2 k}\right] \leq C_{k} L^{k}\left((n+1)^{\theta}-n^{\theta}\right)^{2 k} .
$$

If we choose an integer $k \geq(1-\theta)^{-1}$, as $\left\{(n+1)^{\theta}-n^{\theta}\right\} / n^{\theta-1} \rightarrow \theta$ as $n \rightarrow \infty$, by the Borel-Cantelli lemma we obtain

$$
\limsup _{n \rightarrow \infty} \sup _{n^{\theta} \leq t \leq(n+1)^{\theta}}\left|\int_{n^{\theta}}^{t} \int_{0}^{u} r^{\prime}(u-s) d B(s) d u\right| \leq 1 \quad \text { a.s. }
$$

Gathering the results from (4.3) to (4.7), we see that

$$
\limsup _{n \rightarrow \infty} \sup _{n^{\theta} \leq t \leq(n+1)^{\theta}} \frac{|\tilde{X}(t)|}{\sqrt{2 \log t}} \leq \frac{\Gamma}{\sqrt{\theta}} \quad \text { a.s. }
$$

which implies

$$
\limsup _{t \rightarrow \infty} \frac{|\tilde{X}(t)|}{\sqrt{2 \log t}} \leq \frac{\Gamma}{\sqrt{\theta}} \quad \text { a.s. }
$$

Finally, letting $\theta \rightarrow 1$, we obtain

$$
\limsup _{t \rightarrow \infty} \frac{|X(t)|}{\sqrt{2 \log t}}=\limsup _{t \rightarrow \infty} \frac{|\tilde{X}(t)|}{\sqrt{2 \log t}} \leq \Gamma \quad \text { a.s. }
$$

which is (4.1). We next show that

$$
\limsup _{t \rightarrow \infty} \frac{|X(t)|}{\sqrt{2 \log t}} \geq \Gamma \quad \text { a.s. }
$$

Define the discrete Gaussian process $(\tilde{X}(n))_{n \geq 1}$, where $\tilde{X}(n):=\sigma \int_{0}^{n} r(n-s) d B(s)$. $\tilde{X}(n)$ has variance $v^{2}(n):=\sigma^{2} \int_{0}^{n} r^{2}(s) d s$, so $\left(Z_{n}\right)_{n=1}^{\infty}$ is a sequence of standard normal random variables, where $Z_{n}:=\tilde{X}(n) / v(n)$.

We next prove that there exists a constant $\alpha \in(0,1)$ such that $\left|\operatorname{Cov}\left(Z_{i}, Z_{j}\right)\right| \leq$ $\alpha^{|i-j|}$. To find this constant $\alpha$, let $h \geq 0$ and $n=m+h$. Then

$$
\left|\operatorname{Cov}\left(Z_{m+h}, Z_{m}\right)\right|=\frac{\left|\int_{0}^{m} r(s+h) r(s) d s\right|}{\sqrt{\int_{0}^{m+h} r^{2}(s) d s \int_{0}^{m} r^{2}(s) d s}} .
$$

By the Cauchy-Schwarz inequality

$$
\left|\operatorname{Cov}\left(Z_{m+h}, Z_{m}\right)\right|^{2} \leq \frac{\int_{0}^{m} r^{2}(s+h) d s}{\int_{0}^{m+h} r^{2}(s) d s}=1-\frac{\int_{0}^{h} r^{2}(s) d s}{\int_{0}^{m+h} r^{2}(s) d s} .
$$

Next define $\Gamma_{1}=\int_{0}^{\infty} r^{2}(s) d s$. Then $\int_{0}^{m+h} r^{2}(s) d s \leq \Gamma_{1}$, so

$$
\left|\operatorname{Cov}\left(Z_{m+h}, Z_{m}\right)\right|^{2} \leq 1-\frac{\int_{0}^{h} r^{2}(s) d s}{\int_{0}^{m+h} r^{2}(s) d s} \leq 1-\frac{\int_{0}^{h} r^{2}(s) d s}{\Gamma_{1}}=\frac{\int_{h}^{\infty} r^{2}(s) d s}{\Gamma_{1}}
$$

Now define

$$
\alpha:=\sup _{h \in \mathbb{N}} \alpha(h), \text { where } \alpha(h):=\exp \left[\frac{1}{2 h} \log \frac{\int_{h}^{\infty} r^{2}(s) d s}{\Gamma_{1}}\right] .
$$

Copyright (c) by SIAM. Unauthorized reproduction of this article is prohibited. 
We show that $\alpha \in(0,1)$. Since $r \in L^{1}(0, \infty)$, by (2.6) there exist $C>0$ and $\lambda>0$ such that $|r(t)| \leq C e^{-\lambda t}$ for all $t \geq 0$. Hence

$$
\frac{\int_{h}^{\infty} r^{2}(s) d s}{\Gamma_{1}} \leq \frac{C^{2}}{\Gamma_{1}} \int_{h}^{\infty} e^{-2 \lambda s} d s=\frac{C^{2} e^{-2 \lambda h}}{2 \lambda \Gamma_{1}}
$$

so

$$
\frac{1}{2 h} \log \frac{\int_{h}^{\infty} r^{2}(s) d s}{\Gamma_{1}} \leq-\lambda+\frac{1}{2 h} \log \frac{C^{2}}{2 \lambda \Gamma_{1}} .
$$

Let $\lceil x\rceil$ denote the minimum integer which is greater than $x \in \mathbb{R}$. If $h^{\prime}:=1+$ $\left\lceil(1 / \lambda) \log \left(C^{2} / 2 \lambda \Gamma_{1}\right)\right\rceil$, then for all $h>h^{\prime}$

$$
\frac{\lambda}{2}>\frac{1}{2 h} \log \frac{C^{2}}{2 \lambda \Gamma_{1}} .
$$

Substituting (4.11) into (4.10), we obtain $0<\alpha(h) \leq e^{-\lambda / 2}$ for all $h>h^{\prime}$. For $h<h^{\prime}$, since $r$ is continuous and $r(0)=1, \int_{0}^{h} r^{2}(s) d s>0$ for all $h>0$, and therefore we have that $\int_{h}^{\infty} r^{2}(s) d s<\int_{0}^{\infty} r^{2}(s) d s$ for all $h>0$. This implies $\alpha(h) \in(0,1)$ for all integers $h$ such that $0<h \leq h^{\prime}$, and so $\alpha \in(0,1)$. Therefore

$$
\alpha \geq \exp \frac{1}{2 h} \log \frac{\int_{h}^{\infty} r^{2}(s) d s}{\Gamma_{1}}, \quad h \in \mathbb{N},
$$

which gives

$$
\frac{\int_{h}^{\infty} r^{2}(s) d s}{\Gamma_{1}} \leq \alpha^{2 h}, \quad h \in\{0\} \cup \mathbb{N} .
$$

Combining (4.9) and (4.12), we get $\left|\operatorname{Cov}\left(Z_{n}, Z_{m}\right)\right| \leq \alpha^{|n-m|}$. Thus by Lemma 3,

$$
\lim _{n \rightarrow \infty} \frac{\max _{1 \leq j \leq n} \tilde{X}(n) / v(n)}{\sqrt{2 \log n}}=1 \quad \text { a.s. }
$$

Since Lemma 2 implies

$$
\limsup _{n \rightarrow \infty} \frac{|\tilde{X}(n)| / v(n)}{\sqrt{2 \log n}}=\limsup _{n \rightarrow \infty} \frac{\max _{1 \leq j \leq n}|\tilde{X}(n)| / v(n)}{\sqrt{2 \log n}},
$$

combining these relations gives

$$
\limsup _{n \rightarrow \infty} \frac{|\tilde{X}(n)| / v(n)}{\sqrt{2 \log n}}=1 \quad \text { a.s. }
$$

Therefore

$$
\begin{aligned}
\limsup _{t \rightarrow \infty} \frac{|X(t)|}{\Gamma \sqrt{2 \log t}} & =\limsup _{t \rightarrow \infty} \frac{|\tilde{X}(t)|}{\Gamma \sqrt{2 \log t}} \\
& =\limsup _{t \rightarrow \infty} \frac{|\tilde{X}(t)| / v(t)}{\sqrt{2 \log t}} \geq \limsup _{n \rightarrow \infty} \frac{|\tilde{X}(n)| / v(n)}{\sqrt{2 \log n}},
\end{aligned}
$$

which implies (4.8). Since (4.1) also holds, we have established (3.3). 
It remains to prove (3.4) and (3.5). We prove (3.4). First, note by (3.3) that

$$
\limsup _{t \rightarrow \infty} \frac{X(t)}{\sqrt{2 \log t}} \leq \limsup _{t \rightarrow \infty} \frac{|X(t)|}{\sqrt{2 \log t}}=\Gamma \quad \text { a.s. }
$$

By the definitions of $\tilde{X}, Z$, and $v$, we deduce that

$$
\limsup _{t \rightarrow \infty} \frac{X(t)}{\sqrt{2 \log t}}=\limsup _{t \rightarrow \infty} \frac{\tilde{X}(t)}{\sqrt{2 \log t}} \geq \limsup _{n \rightarrow \infty} \frac{\tilde{X}(n)}{\sqrt{2 \log n}}=\limsup _{n \rightarrow \infty} \frac{Z_{n} v(n)}{\sqrt{2 \log n}} .
$$

Using the fact that $v(n) \rightarrow \Gamma$ as $n \rightarrow \infty$ and Lemmas 2 and 3, we obtain

$$
\begin{aligned}
\limsup _{n \rightarrow \infty} \frac{Z_{n} v(n)}{\sqrt{2 \log n}} & =\limsup _{n \rightarrow \infty} \frac{Z_{n}}{\sqrt{2 \log n}} \cdot \Gamma=\limsup _{n \rightarrow \infty} \frac{\max _{1 \leq j \leq n} Z_{j}}{\sqrt{2 \log n}} \cdot \Gamma \\
& =\lim _{n \rightarrow \infty} \frac{\max _{1 \leq j \leq n} Z_{j}}{\sqrt{2 \log n}} \cdot \Gamma=\Gamma,
\end{aligned}
$$

and so (3.4) holds. Equation (3.5) may be obtained by a symmetric argument.

4.2. Proof of Theorem 2. Let $x$ be the solution of (2.2). Then $x(t) \rightarrow 0$ as $t \rightarrow \infty$, because $v_{0}(\nu)<0$. Then $\tilde{X}(t)=X(t)-x(t)$, where

$$
\tilde{X}(t):=\int_{0}^{t} r(t-s) \Sigma d B(s), \quad t \geq 0 .
$$

Notice that $\tilde{X}(t) \in \mathbb{R}^{d}$ for each $t \geq 0$. Also $\tilde{X}(t)=\int_{0}^{t} \rho(t-s) d B(s), t \geq 0$, where $\rho(t)=r(t) \Sigma$ is a $d \times m$ matrix-valued function in which each entry must obey $\left|\rho_{i j}(t)\right| \leq$ $C e^{-v_{0}(\nu) t / 2}, t \geq 0$, for some $C>0$. Hence $\tilde{X}_{i}(t):=\left\langle X(t), \mathbf{e}_{i}\right\rangle$ obeys

$$
\tilde{X}_{i}(t)=\sum_{j=1}^{m} \int_{0}^{t} \rho_{i j}(t-s) d B_{j}(s), \quad t \geq 0 .
$$

Define $\rho_{i}(t) \geq 0$ with $\rho_{i}^{2}(t)=\sum_{j=1}^{m} \rho_{i j}^{2}(t), t \geq 0$. Then $\tilde{X}_{i}(t)$ is normally distributed with mean 0 and variance $v_{i}(t)=\int_{0}^{t} \rho_{i}^{2}(s) d s$. Since $\rho_{i} \in L^{2}(0, \infty)$, we have that $v_{i}(t) \rightarrow \int_{0}^{\infty} \rho_{i}^{2}(s) d s=\int_{0}^{\infty} \sum_{j=1}^{m} \rho_{i j}^{2}(t) d t=: \sigma_{i}^{2}$ as $t \rightarrow \infty$. Moreover, $\left|\rho_{i}(t)\right| \leq$ $C m e^{-v_{0}(\nu) t / 2}, t \geq 0$. The argument used to prove (4.8) now establishes

$$
\limsup _{t \rightarrow \infty} \frac{\left|\tilde{X}_{i}(t)\right|}{\sqrt{2 \log t}} \geq \sigma_{i} \quad \text { a.s. }
$$

We now wish to prove

$$
\limsup _{t \rightarrow \infty} \frac{\left|\tilde{X}_{i}(t)\right|}{\sqrt{2 \log t}} \leq \sigma_{i} \text { a.s. }
$$

We first note for each $\theta>0$ that the argument used to prove (4.3) can be used to establish

$$
\limsup _{n \rightarrow \infty} \frac{\left|\tilde{X}_{i}\left(n^{\theta}\right)\right|}{\sqrt{2 \log \left(n^{\theta}\right)}} \leq \sqrt{\frac{\sigma_{i}^{2}}{\theta}} \quad \text { a.s. }
$$

Copyright $\odot$ by SIAM. Unauthorized reproduction of this article is prohibited. 
In a manner similar to (4.4), we can rewrite $\tilde{X}$ according to

$$
\begin{aligned}
\tilde{X}_{i}(t) & =\sum_{j=1}^{m} \int_{0}^{t}\left(\rho_{i j}(0)+\int_{0}^{t-s} \rho_{i j}^{\prime}(u) d u\right) d B_{j}(s) \\
& =\sum_{j=1}^{m} \rho_{i j}(0) B_{j}(t)+\sum_{j=1}^{m} \int_{0}^{t} \int_{0}^{u} \rho_{i j}^{\prime}(u-s) d B_{j}(s) d u .
\end{aligned}
$$

Hence for $t \in\left[n^{\theta},(n+1)^{\theta}\right]$ we get

$$
\tilde{X}_{i}(t)-\tilde{X}_{i}\left(n^{\theta}\right)=\sum_{j=1}^{m} \rho_{i j}(0)\left(B_{j}(t)-B_{j}\left(n^{\theta}\right)\right)+\sum_{j=1}^{m} \int_{n^{\theta}}^{t} \int_{0}^{u} \rho_{i j}^{\prime}(u-s) d B_{j}(s) d u,
$$

which implies

$$
\sup _{n^{\theta} \leq t \leq(n+1)^{\theta}}\left|\tilde{X}_{i}(t)-\tilde{X}_{i}\left(n^{\theta}\right)\right| \leq \sum_{j=1}^{m}\left|\rho_{i j}(0)\right| \sup _{n^{\theta} \leq t \leq(n+1)^{\theta}}\left|B_{j}(t)-B_{j}\left(n^{\theta}\right)\right|+\sum_{j=1}^{m} U_{n}^{(i, j)},
$$

where we have defined

$$
U_{n}^{(i, j)}=\sup _{n^{\theta} \leq t \leq(n+1)^{\theta}}\left|\int_{n^{\theta}}^{t} \int_{0}^{u} \rho_{i j}^{\prime}(u-s) d B_{j}(s) d u\right| .
$$

Then, using the technique used to prove (4.7), we can show that

$$
\limsup _{n \rightarrow \infty} U_{n}^{(i, j)} \leq 1 \quad \text { a.s. }
$$

By (4.6), we have

$$
\limsup _{n \rightarrow \infty} \sup _{n^{\theta} \leq t \leq(n+1)^{\theta}}\left|B_{j}(t)-B_{j}\left(n^{\theta}\right)\right| \leq 1 \quad \text { a.s. }
$$

Therefore,

$$
\limsup _{n \rightarrow \infty} \frac{\sup _{n^{\theta} \leq t \leq(n+1)^{\theta}}\left|\tilde{X}_{i}(t)-\tilde{X}_{i}\left(n^{\theta}\right)\right|}{\sqrt{2 \log n^{\theta}}}=0 \text { a.s. }
$$

Using this estimate and (4.16), we obtain

$$
\limsup _{n \rightarrow \infty} \sup _{n^{\theta} \leq t \leq(n+1)^{\theta}} \frac{\left|\tilde{X}_{i}(t)\right|}{\sqrt{2 \log t}} \leq \sqrt{\frac{\sigma_{i}^{2}}{\theta}} \text { a.s. }
$$

which implies

$$
\limsup _{t \rightarrow \infty} \frac{\left|\tilde{X}_{i}(t)\right|}{\sqrt{2 \log t}} \leq \sqrt{\frac{\sigma_{i}^{2}}{\theta}} \quad \text { a.s. }
$$

Letting $\theta \rightarrow 1$ through the rational numbers implies (4.15). Combining (4.14) and (4.15) yields

$$
\limsup _{t \rightarrow \infty} \frac{\left|\tilde{X}_{i}(t)\right|}{\sqrt{2 \log t}} \leq \sigma_{i} \quad \text { a.s. }
$$

Proceeding as at the end of Theorem 1, we can also establish (3.7).

Copyright (c) by SIAM. Unauthorized reproduction of this article is prohibited. 
To prove (3.9), note that there is an $i^{*} \in\{1, \ldots, d\}$ such that $\sigma_{i^{*}}=\max _{1 \leq i \leq d} \sigma_{i}$. Next, note for each $t \geq 0$ that

$$
\max _{1 \leq i \leq d}\left|X_{i}(t)\right|=\max \left(\left|X_{1}(t)\right|,\left|X_{2}(t)\right|, \ldots,\left|X_{i^{*}}(t)\right|, \ldots,\left|X_{d}(t)\right|\right) \geq\left|X_{i^{*}}(t)\right| .
$$

Hence

$$
\limsup _{t \rightarrow \infty} \frac{\max _{1 \leq i \leq d}\left|X_{i}(t)\right|}{\sqrt{2 \log t}} \geq \limsup _{t \rightarrow \infty} \frac{\left|X_{i^{*}}(t)\right|}{\sqrt{2 \log t}}=\sigma_{i^{*}}=\max _{i=1, \ldots, d} \sigma_{i} \quad \text { a.s. }
$$

Let $p$ be an integer greater than unity. Note that $\max _{1 \leq i \leq d}\left|x_{i}\right| \leq\left(\sum_{i=1}^{d}\left|x_{i}\right|^{p}\right)^{1 / p}$, so we have

$$
\begin{aligned}
\left(\limsup _{t \rightarrow \infty} \frac{\max _{1 \leq i \leq d}\left|X_{i}(t)\right|}{\sqrt{2 \log t}}\right)^{p} & =\limsup _{t \rightarrow \infty} \frac{\left(\max _{1 \leq i \leq d}\left|X_{i}(t)\right|\right)^{p}}{(\sqrt{2 \log t})^{p}} \\
& \leq \limsup _{t \rightarrow \infty} \frac{\sum_{i=1}^{d}\left|X_{i}(t)\right|^{p}}{(\sqrt{2 \log t})^{p}} \\
& \leq \sum_{i=1}^{d} \limsup _{t \rightarrow \infty} \frac{\left|X_{i}(t)\right|^{p}}{(\sqrt{2 \log t})^{p}} \\
& =\sum_{i=1}^{d}\left(\limsup _{t \rightarrow \infty} \frac{\left|X_{i}(t)\right|}{\sqrt{2 \log t}}\right)^{p}=\sum_{i=1}^{d} \sigma_{i}^{p} .
\end{aligned}
$$

Hence

$$
\limsup _{t \rightarrow \infty} \frac{\max _{1 \leq i \leq d}\left|X_{i}(t)\right|}{\sqrt{2 \log t}} \leq\left(\sum_{i=1}^{d} \sigma_{i}^{p}\right)^{1 / p} \quad \text { a.s. }
$$

Letting $p \rightarrow \infty$ through the natural numbers yields

$$
\limsup _{t \rightarrow \infty} \frac{\max _{1 \leq i \leq d}\left|X_{i}(t)\right|}{\sqrt{2 \log t}} \leq \max _{1 \leq i \leq d} \sigma_{i} \quad \text { a.s. }
$$

since $\left(\sum_{i=1}^{d} \sigma_{i}^{p}\right)^{1 / p} \rightarrow \max _{1 \leq i \leq d} \sigma_{i}$ as $p \rightarrow \infty$. Combining (4.18) and (4.19) yields (3.9).

4.3. Proof of Theorem 3. By (3.11), for any $\phi \in C\left([-\tau, 0] ; \mathbb{R}^{d}\right)$ and $\varepsilon>0$, there exists $L(\varepsilon)>0$ such that (3.13) holds, viz.,

$$
|N(t, \phi)|_{2} \leq L(\varepsilon)+\varepsilon\|\phi\|_{2} \quad \text { for all }(t, \phi) \in(0, \infty) \times C\left([-\tau, 0] ; \mathbb{R}^{d}\right) .
$$

Choose $\varepsilon \int_{0}^{\infty}|r(s)|_{2} d s<1 / 2$. Suppose $Y$ obeys (2.7) with $Y(t)=\phi(t)=X(t)$ for $t \in$ $[-\tau, 0]$. Define $Z$ by $Z(t)=X(t)-Y(t)$ for $t \geq-\tau$. Then $Z^{\prime}(t)=L\left(Z_{t}\right)+N\left(t, Y_{t}+Z_{t}\right)$ for $t>0$ and $Z(t)=0$ for $t \in[-\tau, 0]$. Hence

$$
Z(t)=\int_{0}^{t} r(t-s) N\left(s, Y_{s}+Z_{s}\right) d s, \quad t \geq 0 .
$$

Therefore for $t \geq 0$

$$
|Z(t)|_{2} \leq \int_{0}^{t}|r(t-s)|_{2}\left\{L(\varepsilon)+\varepsilon \sup _{s-\tau \leq u \leq s}|Y(u)+Z(u)|_{2}\right\} d s .
$$

Copyright (c) by SIAM. Unauthorized reproduction of this article is prohibited. 
Hence, with $f_{\varepsilon}(t):=L(\varepsilon) \int_{0}^{\infty}|r(s)|_{2} d s+\varepsilon \int_{0}^{t}|r(t-s)|_{2} \sup _{s-\tau \leq u \leq s}|Y(u)|_{2} d s$, we have

$$
\begin{aligned}
|Z(t)|_{2} & \leq f_{\varepsilon}(t)+\varepsilon \int_{0}^{t}|r(t-s)|_{2} \sup _{s-\tau \leq u \leq s}|Z(u)|_{2} d s \\
& \leq f_{\varepsilon}(t)+\varepsilon \int_{0}^{t}|r(t-s)|_{2}\left(\sup _{-\tau \leq u \leq 0}|Z(u)|_{2}+\sup _{0 \leq u \leq s}|Z(u)|_{2}\right) d s \\
& \leq f_{\varepsilon}(t)+\varepsilon \int_{0}^{t}|r(t-s)|_{2} \sup _{0 \leq u \leq s}|Z(u)|_{2} d s .
\end{aligned}
$$

Now, define $Z^{*}(t)=\sup _{0 \leq s \leq t}|Z(s)|_{2}, f_{\varepsilon}^{*}(T)=\sup _{0 \leq t \leq T} f_{\varepsilon}(t)$. Then, for $T>0$, we have

$$
Z^{*}(T)=\sup _{0 \leq t \leq T}|Z(t)|_{2} \leq f_{\varepsilon}^{*}(T)+\varepsilon \sup _{0 \leq t \leq T} \int_{0}^{t}|r(t-s)|_{2} Z^{*}(s) d s .
$$

Now $Z^{*}(s) \leq Z^{*}(t)$ for $0 \leq s \leq t$, so as $r \in L^{1}(0, \infty)$ we get

$$
\begin{aligned}
\varepsilon \sup _{0 \leq t \leq T} \int_{0}^{t}|r(t-s)|_{2} Z^{*}(s) d s & \leq \varepsilon \sup _{0 \leq t \leq T} Z^{*}(t) \int_{0}^{t}|r(t-s)|_{2} d s \\
& =\varepsilon \sup _{0 \leq t \leq T} Z^{*}(t) \int_{0}^{t}|r(s)|_{2} d s \\
& \leq \varepsilon \sup _{0 \leq t \leq T} Z^{*}(t) \int_{0}^{\infty}|r(s)|_{2} d s \\
& \leq \varepsilon \int_{0}^{\infty}|r(s)|_{2} d s \sup _{0 \leq t \leq T} Z^{*}(t) \\
& =\varepsilon \int_{0}^{\infty}|r(s)|_{2} d s \cdot Z^{*}(T) .
\end{aligned}
$$

Inserting this into (4.20) gives

$$
Z^{*}(T) \leq f_{\varepsilon}^{*}(T)+\varepsilon Z^{*}(T) \int_{0}^{\infty}|r(s)|_{2} d s \leq f_{\varepsilon}^{*}(T)+\frac{1}{2} Z^{*}(T) .
$$

Hence $Z^{*}(t) \leq 2 f_{\varepsilon}^{*}(t)$ for all $t \geq 0$. Now, recall that

$$
f_{\varepsilon}(t)=L(\varepsilon) \int_{0}^{\infty}|r(s)|_{2} d s+\varepsilon \int_{0}^{t}|r(t-s)|_{2} \sup _{s-\tau \leq u \leq s}|Y(u)|_{2} d s,
$$

so as $Y(t)=\phi(t)$ for $t \in[-\tau, 0]$, and setting $Y^{*}(t)=\sup _{0 \leq s \leq t}|Y(s)|_{2}$, we get

$$
f_{\varepsilon}(t) \leq L(\varepsilon) \int_{0}^{\infty}|r(s)|_{2} d s+\varepsilon \int_{0}^{t}|r(t-s)|_{2}\left(\sup _{-\tau \leq u \leq 0}|\phi(u)|_{2}+\sup _{0 \leq u \leq s}|Y(u)|_{2}\right) d s
$$

so

$$
\begin{array}{r}
f_{\varepsilon}(t) \leq\left(L(\varepsilon)+\varepsilon \sup _{-\tau \leq u \leq 0}|\phi(u)|_{2}\right) \int_{0}^{\infty}|r(s)|_{2} d s \\
+\varepsilon \int_{0}^{t}|r(t-s)|_{2} Y^{*}(s) d s .
\end{array}
$$

Copyright (c) by SIAM. Unauthorized reproduction of this article is prohibited. 
Now, by Lemma 5, we get

$$
\limsup _{t \rightarrow \infty} \frac{Y^{*}(t)}{\sqrt{2 \log t}}=\limsup _{t \rightarrow \infty} \frac{|Y(t)|_{2}}{\sqrt{2 \log t}} .
$$

We already know from Theorem 2 that there is a $c_{0}>0$ such that

$$
\limsup _{t \rightarrow \infty} \frac{|Y(t)|_{\infty}}{\sqrt{2 \log t}}=c_{0} \quad \text { a.s. }
$$

so by norm equivalence there is a deterministic $c_{1}>0$ such that

$$
\limsup _{t \rightarrow \infty} \frac{|Y(t)|_{2}}{\sqrt{2 \log t}} \leq c_{1} \quad \text { a.s. }
$$

Hence by Lemma 5 we obtain

$$
\limsup _{t \rightarrow \infty} \frac{Y^{*}(t)}{\sqrt{2 \log t}} \leq c_{1} \quad \text { a.s. }
$$

Therefore, by Lemma 4, we have

$$
\limsup _{t \rightarrow \infty} \frac{1}{\sqrt{2 \log t}} \int_{0}^{t}|r(t-s)|_{2} Y^{*}(s) d s \leq c_{1} \int_{0}^{\infty}|r(s)|_{2} d s \quad \text { a.s. }
$$

And so, from (4.21), we get

$$
\limsup _{t \rightarrow \infty} \frac{f_{\varepsilon}(t)}{\sqrt{2 \log t}} \leq \varepsilon c_{1} \int_{0}^{\infty}|r(s)|_{2} d s \quad \text { a.s. }
$$

By Lemma 5, this implies that

$$
\limsup _{t \rightarrow \infty} \frac{f_{\varepsilon}^{*}(t)}{\sqrt{2 \log t}} \leq \varepsilon c_{1} \int_{0}^{\infty}|r(s)|_{2} d s \quad \text { a.s. }
$$

Recalling that $Z^{*}(t) \leq 2 f_{\varepsilon}^{*}(t)$ for all $t \geq 0$, we have

$$
\limsup _{t \rightarrow \infty} \frac{Z^{*}(t)}{\sqrt{2 \log t}} \leq 2 \varepsilon c_{1} \int_{0}^{\infty}|r(s)|_{2} d s \quad \text { a.s. }
$$

Perusal of the above proof shows that the almost sure event $\left(\Omega^{*}\right.$ say) on which (4.22) holds is independent of $\varepsilon$, where $\varepsilon<(1 / 2)\left(\int_{0}^{\infty}|r(s)|_{2} d s\right)^{-1}$. Therefore, for each $\omega \in \Omega^{*}$, we may let $\varepsilon \rightarrow 0^{+}$to obtain

$$
\limsup _{t \rightarrow \infty} \frac{Z^{*}(t, \omega)}{\sqrt{2 \log t}}=0
$$

Copyright (c) by SIAM. Unauthorized reproduction of this article is prohibited. 
Hence

$$
\limsup _{t \rightarrow \infty} \frac{Z^{*}(t)}{\sqrt{2 \log t}}=0 \quad \text { a.s. }
$$

That is,

$$
\lim _{t \rightarrow \infty} \frac{|X(t)-Y(t)|_{2}}{\sqrt{2 \log t}}=0 \quad \text { a.s. }
$$

and, moreover,

$$
\lim _{t \rightarrow \infty} \frac{\left|X_{i}(t)-Y_{i}(t)\right|}{\sqrt{2 \log t}}=0 \quad \text { a.s. }
$$

Now, it is known from Theorem 2 that

$$
\limsup _{t \rightarrow \infty} \frac{\left|Y_{i}(t)\right|}{\sqrt{2 \log t}}=\sigma_{i} \quad \text { a.s. }
$$

where $\sigma_{i}$ is given by (3.8). Thus

$$
\limsup _{t \rightarrow \infty} \frac{\left|X_{i}(t)\right|}{\sqrt{2 \log t}} \leq \limsup _{t \rightarrow \infty} \frac{\left|Y_{i}(t)\right|}{\sqrt{2 \log t}}+\limsup _{t \rightarrow \infty} \frac{\left|X_{i}(t)-Y_{i}(t)\right|}{\sqrt{2 \log t}}=\sigma_{i} \quad \text { a.s. }
$$

Similarly

$$
\begin{aligned}
\limsup _{t \rightarrow \infty} \frac{\left|X_{i}(t)\right|}{\sqrt{2 \log t}} & \geq \limsup _{t \rightarrow \infty}\left(\frac{\left|Y_{i}(t)\right|}{\sqrt{2 \log t}}-\frac{\left|X_{i}(t)-Y_{i}(t)\right|}{\sqrt{2 \log t}}\right) \\
& =\limsup _{t \rightarrow \infty} \frac{\left|Y_{i}(t)\right|}{\sqrt{2 \log t}}=\sigma_{i} \quad \text { a.s. }
\end{aligned}
$$

Combining these inequalities, we get

$$
\limsup _{t \rightarrow \infty} \frac{\left|X_{i}(t)\right|}{\sqrt{2 \log t}}=\sigma_{i} \quad \text { a.s. }
$$

Write $X_{i}(t)=X_{i}(t)-Y_{i}(t)+Y_{i}(t)$. Using the fact that

$$
\limsup _{t \rightarrow \infty} \frac{Y_{i}(t)}{\sqrt{2 \log t}}=\sigma_{i} \quad \text { a.s. }
$$

by (3.7), together with (4.23), we get the first part of (3.15). One proceeds similarly to the end of Theorem 1 to obtain the second part of (3.15). We may proceed as in the proof of Theorem 2 to show that these limits imply

$$
\limsup _{t \rightarrow \infty} \frac{|X(t)|_{\infty}}{\sqrt{2 \log t}}=\max _{1 \leq i \leq d} \sigma_{i} \quad \text { a.s. }
$$

proving the result. 


\section{Auxiliary results.}

5.1. Proof of Lemma 2. Define

$$
L=\limsup _{n \rightarrow \infty} \frac{a_{n}}{\gamma(n)}, \quad M:=\limsup _{n \rightarrow \infty} \frac{\max _{1 \leq j \leq n} a_{j}}{\gamma(n)} .
$$

Then $L \leq M$. Since $\limsup _{n \rightarrow \infty} a_{n} \geq 0$ and $\gamma$ is positive, $L \geq 0$. In the case that $L=+\infty$, we automatically have $M=+\infty$. Therefore we focus on the case where $L \in[0, \infty)$.

For every $\varepsilon>0$ there exists $N_{1}(\varepsilon) \in \mathbb{N}$ such that $a_{n} / \gamma(n) \leq L+\varepsilon$ for all $n \geq N_{1}(\varepsilon)$. Define $A(\varepsilon)=\max _{1 \leq j \leq N_{1}(\varepsilon)} a_{j}$. Since $\gamma(n) \rightarrow \infty$ as $n \rightarrow \infty$, there exists $N_{2}(\varepsilon) \in \mathbb{N}$ such that $\gamma(n)(L+\varepsilon)>A(\varepsilon)$ for all $n \geq N_{2}(\varepsilon)$. Now, let $n \geq N_{1}(\varepsilon)+1$. Then, as $\gamma$ is nondecreasing, we have

$$
\begin{aligned}
\max _{1 \leq j \leq n} a_{j} & =\max \left(A(\varepsilon), \max _{N_{1}(\varepsilon)+1 \leq j \leq n} a_{j}\right) \\
& \leq \max \left(A(\varepsilon), \max _{N_{1}(\varepsilon)+1 \leq j \leq n} \gamma(j)(L+\varepsilon)\right) \\
& =\max (A(\varepsilon), \gamma(n)(L+\varepsilon)) .
\end{aligned}
$$

Define $N(\varepsilon)=\max \left(N_{1}(\varepsilon)+1, N_{2}(\varepsilon)\right)$. Let $n \geq N(\varepsilon)$. Then $\gamma(n)(L+\varepsilon)>A(\varepsilon)$ and also $\max _{1 \leq j \leq n} a_{j} \leq \max (A(\varepsilon), \gamma(n)(L+\varepsilon))$, so

$$
\max _{1 \leq j \leq n} a_{j} \leq \max (A(\varepsilon), \gamma(n)(L+\varepsilon))=\gamma(n)(L+\varepsilon) \quad \text { for all } n \geq N(\varepsilon) .
$$

Since $\varepsilon>0$ is arbitrary, we therefore have

$$
\limsup _{n \rightarrow \infty} \frac{\max _{1 \leq j \leq n} a_{j}}{\gamma(n)} \leq L,
$$

or $M \leq L$. But since $L \leq M$, we have $M=L$ in the case when $L \geq 0$ is finite and hence the result.

5.2. Proof of Lemma 4. Without loss of generality, let $\int_{0}^{\infty} \kappa(s) d s=1$. For every $\varepsilon>0$, there is $T=T(\varepsilon)>0$ such that $\int_{T}^{\infty} \kappa(s) d s<\varepsilon$. For $t \geq T$, we have

$$
\begin{aligned}
\frac{(\kappa * \vartheta)(t)}{\vartheta(t)}-\int_{0}^{t} \kappa(s) d s=\int_{0}^{T} \kappa(s) & \left(\frac{\vartheta(t-s)}{\vartheta(t)}-1\right) d s \\
& +\int_{T}^{t} \kappa(s)\left(\frac{\vartheta(t-s)}{\vartheta(t)}-1\right) d s
\end{aligned}
$$

Now, as $\int_{0}^{\infty} \kappa(s) d s=1$ and $\vartheta$ is an increasing function,

$$
\left|\int_{0}^{T} \kappa(s)\left(\frac{\vartheta(t-s)}{\vartheta(t)}-1\right) d s\right| \leq 1-\frac{\vartheta(t-T)}{\vartheta(t)} .
$$

Moreover, as $\kappa$ is nonnegative and $\vartheta$ is increasing, we have

$$
\left|\int_{T}^{t} \kappa(s)\left(\frac{\vartheta(t-s)}{\vartheta(t)}-1\right) d s\right|=\int_{T}^{t} \kappa(s)\left(1-\frac{\vartheta(t-s)}{\vartheta(t)}\right) d s \leq \int_{T}^{\infty} \kappa(s) d s .
$$

Copyright (c) by SIAM. Unauthorized reproduction of this article is prohibited. 
Thus

$$
\begin{aligned}
\left|\int_{0}^{t} \frac{\kappa(s) \vartheta(t-s)}{\vartheta(t)} d s-\int_{0}^{t} \kappa(s) d s\right| & \leq 1-\frac{\vartheta(t-T)}{\vartheta(t)}+\int_{T}^{\infty} \kappa(s) d s \\
& <1-\frac{\vartheta(t-T)}{\vartheta(t)}+\varepsilon .
\end{aligned}
$$

Using $\vartheta(t-T) / \vartheta(t) \rightarrow 1$ as $t \rightarrow \infty$ and then letting $\varepsilon \rightarrow 0$ yields the result.

5.3. Proof of Lemma 3. Define $\Phi(x)=\frac{1}{\sqrt{2 \pi}} \int_{-\infty}^{x} e^{-u^{2} / 2} d u$. Mill's estimate tells us that

$$
1-\Phi(x) \leq \frac{1}{\sqrt{2 \pi}} \frac{1}{x} e^{-\frac{x^{2}}{2}}, \quad x>0
$$

Indeed, we also have

$$
\lim _{x \rightarrow \infty} \frac{1-\Phi(x)}{\frac{1}{\sqrt{2 \pi}} \frac{1}{x} e^{-\frac{x^{2}}{2}}}=1 .
$$

In order to prove Lemma 3, we need some existing results about the fluctuations of stationary Gaussian sequences. First, we state the normal comparison lemma.

Lemma 6. Suppose $\left(X_{n}\right)_{n=1}^{\infty}$ is a stationary sequence of standard normal variables. If $\left(r_{h}\right)_{h \geq 0}$ is the autocorrelation function of $X$ so that $\operatorname{Corr}\left(X_{i}, X_{j}\right)=r_{|i-j|}$ and

$$
M_{n}=\max _{1 \leq j \leq n} X_{j}
$$

then for any real sequence $\left(u_{n}\right)_{n \geq 1}$ we have

$$
\left|\mathbb{P}\left[M_{n} \leq u_{n}\right]-\Phi\left(u_{n}\right)^{n}\right| \leq K n \sum_{h=1}^{n}\left|r_{h}\right| \exp \left(-\frac{u_{n}^{2}}{1+\left|r_{h}\right|}\right),
$$

where $K$ depends on $\delta=\sup _{h \geq 1}\left|r_{h}\right|<1$.

The proof of this result may be found in Leadbetter, Lindgren, and Rootzén [35, pp. 81-85]. Another result related to the normal comparison lemma is the following, also given in $[35$, p. 84$]$.

Lemma 7 . Let $X_{j}, Y_{j}$ for $j=1, \ldots, n$ be sequences of standard normal random variables which satisfy

$$
\operatorname{Cov}\left(X_{i}, X_{j}\right) \leq \operatorname{Cov}\left(Y_{i}, Y_{j}\right)
$$

for all $i, j=1, \ldots, n$. If $M_{n}^{X}=\max _{1 \leq j \leq n} X_{j}$ and $M_{n}^{Y}=\max _{1 \leq j \leq n} Y_{j}$, then

$$
\mathbb{P}\left[M_{n}^{X} \leq u\right] \leq \mathbb{P}\left[M_{n}^{Y} \leq u\right]
$$

for all $u \in \mathbb{R}$.

We require the following results about sequences of identically distributed normal random variables. The first result is elementary but allows us to refer to appropriate estimates.

Copyright $@$ by SIAM. Unauthorized reproduction of this article is prohibited. 
LEMma 8. If $\left(X_{n}\right)_{n=1}^{\infty}$ is a sequence of jointly normal standard random variables, then

$$
\limsup _{n \rightarrow \infty} \frac{\left|X_{n}\right|}{\sqrt{2 \log n}} \leq 1 \quad \text { a.s. }
$$

Moreover,

$$
\limsup _{n \rightarrow \infty} \frac{\max _{1 \leq j \leq n} X_{j}}{\sqrt{2 \log n}} \leq 1 \quad \text { a.s. }
$$

Proof. For every $\epsilon>0$, Mill's estimate gives

$$
\mathbb{P}\left[\left|X_{n}\right|>\sqrt{2(1+\epsilon) \log n]} \leq \frac{2}{\sqrt{2 \pi}} \frac{1}{\sqrt{2(1+\epsilon) \log n}} \frac{1}{n^{1+\epsilon}},\right.
$$

so by the Borel-Cantelli lemma, for each $\epsilon>0$, we have

$$
\limsup _{n \rightarrow \infty} \frac{\left|X_{n}\right|}{\sqrt{2(1+\epsilon) \log n}} \leq 1 \quad \text { a.s. }
$$

By letting $\epsilon \rightarrow 0$ through rational numbers we get (5.3). Moreover,

$$
\limsup _{n \rightarrow \infty} \frac{\max _{1 \leq j \leq n} X_{j}}{\sqrt{2 \log n}} \leq \limsup _{n \rightarrow \infty} \frac{\max _{1 \leq j \leq n}\left|X_{j}\right|}{\sqrt{2 \log n}}=\limsup _{n \rightarrow \infty} \frac{\left|X_{n}\right|}{\sqrt{2 \log n}} \leq 1 \quad \text { a.s. }
$$

where we have used Lemma 2 at the penultimate step.

We will now use Lemma 6 to obtain the following useful estimate on the maxima of exponentially correlated sequences.

LEMMA 9. Let $\left(X_{n}\right)_{n=1}^{\infty}$ be a stationary sequence of standard normal random variables with $\operatorname{Cov}\left(X_{i}, X_{j}\right)=\lambda^{|i-j|}$ for some $\lambda \in(0,1)$. If $\alpha>4$, then

$$
\sum_{n=2}^{\infty} \mathbb{P}\left[\frac{\max _{1 \leq j \leq n} X_{j}}{\sqrt{\log n}}>\sqrt{\alpha}\right]<\infty .
$$

Proof. Define $u_{n}=\sqrt{\alpha \log n}$ and $\Psi(x)=1-\Phi(x)$. By Mill's estimate we therefore have

$$
1-\Phi\left(u_{n}\right)^{n}=1-\left(1-\Psi\left(u_{n}\right)\right)^{n} \leq n \Psi\left(u_{n}\right) \leq \frac{1}{\sqrt{2 \pi}} \frac{1}{\sqrt{\alpha \log n}} \frac{1}{n^{\frac{\alpha}{2}-1}} .
$$

If $h:=|i-j|$, define $r_{h}=\lambda^{h}$ and $B_{n}=K n \sum_{h=1}^{n}\left|r_{h}\right| \exp \left(\frac{-u_{n}^{2}}{1+\left|r_{h}\right|}\right)$. From Lemma 6 we have

$$
\mathbb{P}\left[M_{n}>u_{n}\right] \leq 1-\Phi\left(u_{n}\right)^{n}+B_{n} .
$$

With the choice of $u_{n}$ we easily obtain, for some $0<K_{2}<\infty$,

$$
B_{n} \leq K \frac{1}{n^{\frac{\alpha}{2}-1}} \sum_{h=1}^{n}\left|r_{h}\right|<\frac{K_{2}}{n^{\frac{\alpha}{2}-1}} .
$$

Copyright $@$ by SIAM. Unauthorized reproduction of this article is prohibited. 
Taking (5.6), (5.7), and (5.8) together we see that there exist $0<K_{1}<\infty$ and $N \in \mathbb{N}$ such that for all $n \geq N$

$$
\mathbb{P}\left[M_{n}>\sqrt{\alpha \log n}\right] \leq K_{1} \frac{1}{\sqrt{\alpha \log n}} \frac{1}{n^{\frac{\alpha}{2}-1}}+K_{2} \frac{1}{n^{\frac{\alpha}{2}-1}},
$$

from which (5.5) follows.

We are now in a position to prove Lemma 3.

Proof. In Lemma 8, we have already established

$$
\limsup _{n \rightarrow \infty} \frac{\max _{1 \leq j \leq n} X_{j}}{\sqrt{2 \log n}} \leq 1 \quad \text { a.s. }
$$

Therefore, it suffices to prove

$$
\liminf _{n \rightarrow \infty} \frac{\max _{1 \leq j \leq n} X_{j}}{\sqrt{2 \log n}} \geq 1 \quad \text { a.s. }
$$

To do this let $Y=\left(Y_{n}\right)_{n \geq 1}$ be a sequence of jointly normal standard random variables satisfying $\operatorname{Cov}\left(Y_{i}, Y_{j}\right)=\bar{\lambda}^{|i-j|}$. The sequence $Y$ has two important properties. First, it dominates $X$ in the sense made precise by Lemma 7 ; the second vital property is motivated presently. To see that $Y$ dominates $X$, let $M_{n}^{X}, M_{n}^{Y}$ have the same meaning as in Lemma 7. Thus, for all $\varepsilon>0$, we have by Lemma 7

$$
\mathbb{P}\left[M_{n}^{X} \leq(1-\varepsilon) \sqrt{2 \log n}\right] \leq \mathbb{P}\left[M_{n}^{Y} \leq(1-\varepsilon) \sqrt{2 \log n}\right] .
$$

The proof is thus complete (after taking $\varepsilon \downarrow 0$ ) by the Borel-Cantelli lemma if we can show

$$
\sum_{n=1}^{\infty} \mathbb{P}\left[M_{n}^{Y} \leq(1-\varepsilon) \sqrt{2 \log n}\right]<\infty
$$

The second important property of the auxiliary process $Y$ is that the exactly geometric decay in the autocovariance function enables us to construct (via Gram-Schmidt orthonormalization) a sequence of independent Gaussian random variables, whose large fluctuations are then readily determined by use of both Borel-Cantelli lemmata.

The remainder of the proof is devoted to demonstrating (5.9). For all $\varepsilon \in(0,1)$ there exists $k_{1}(\varepsilon) \in \mathbb{N}$ such that for all $k>k_{1}(\varepsilon)$

$$
\lambda^{k} \leq \frac{2}{3}\left(\sqrt{9+8 \varepsilon-4 \varepsilon^{2}}-3(1-\varepsilon)\right)=: \alpha(\varepsilon)>0 .
$$

Fix $k=k_{1}(\varepsilon)+1$. Note that $\alpha(\varepsilon)$ is the unique positive root of $f_{\varepsilon}(x)=-2 \varepsilon+\varepsilon^{2}+$ $3 x(1-\varepsilon)+\frac{13}{4} x^{2}$, so we have

$$
\mu:=\frac{1-\varepsilon+\frac{3}{2} \lambda^{k}}{\sqrt{1-\lambda^{2 k}}} \in(0,1) .
$$

We now seek a bound on $\mathbb{P}\left[\max _{1 \leq j \leq N} Y_{j+k} \leq(1-\varepsilon) \sqrt{2 \log N}\right]$. To do this, we notice that the integer $n$ is well defined by $n k \leq N<(n+1) k$. Then

$$
\begin{aligned}
\frac{\max _{1 \leq j \leq N} Y_{j+k}}{\sqrt{2 \log N}} & \geq \frac{\max _{1 \leq j \leq n k} Y_{j+k}}{\sqrt{2 \log N}} \\
& \geq \frac{\max _{1 \leq j \leq n k} Y_{j+k}-\lambda^{k} Y_{j}}{\sqrt{2 \log N}}-\lambda^{k} \frac{\max _{1 \leq j \leq n k}-Y_{j}}{\sqrt{2 \log N}} .
\end{aligned}
$$

Copyright (c) by SIAM. Unauthorized reproduction of this article is prohibited. 
We now use the observation that $\mathbb{P}[U+V \geq u] \leq \mathbb{P}[U \geq v]+\mathbb{P}[V \geq u-v]$ for any random variables $U$ and $V$ and constants $u$ and $v$ to obtain the bound

$$
\begin{aligned}
& \mathbb{P}\left[\frac{\max _{1 \leq j \leq N} Y_{j+k}}{\sqrt{2 \log N}} \leq 1-\varepsilon\right] \\
& \leq \mathbb{P}\left[\frac{\max _{1 \leq j \leq n k} Y_{j+k}-\lambda^{k} Y_{j}}{\sqrt{2 \log N}}-\lambda^{k} \frac{\max _{1 \leq j \leq n k}-Y_{j}}{\sqrt{2 \log N}} \leq 1-\varepsilon\right] \\
& \leq \mathbb{P}\left[\lambda^{k} \frac{\max _{1 \leq j \leq n k}-Y_{j}}{\sqrt{2 \log N}} \geq \frac{3}{2} \lambda^{k}\right]+\mathbb{P}\left[-\frac{\max _{1 \leq j \leq n k} Y_{j+k}-\lambda^{k} Y_{j}}{\sqrt{2 \log N}} \geq \varepsilon-1-\frac{3}{2} \lambda^{k}\right] \\
& =\mathbb{P}\left[\frac{\max _{1 \leq j \leq n k} Y_{j}}{\sqrt{2 \log N}} \geq \frac{3}{2}\right]+\mathbb{P}\left[\frac{\max _{1 \leq j \leq n k} Y_{j+k}-\lambda^{k} Y_{j}}{\sqrt{2 \log N}} \leq 1-\varepsilon+\frac{3}{2} \lambda^{k}\right] \\
& \leq \mathbb{P}\left[\frac{\max _{1 \leq j \leq N} Y_{j}}{\sqrt{2 \log N}} \geq \frac{3}{2}\right]+\mathbb{P}\left[\frac{\max _{1 \leq j \leq n k} Y_{j+k}-\lambda^{k} Y_{j}}{\sqrt{2 \log N}} \leq 1-\varepsilon+\frac{3}{2} \lambda^{k}\right],
\end{aligned}
$$

where we exploit the symmetry of distribution of the random variable $Y_{j}$ at the penultimate step, and $n k \leq N$ at the last step. We now wish to show that both terms on the right-hand side of (5.11) are summable over $N$. For the first term, we note that the estimate (5.5) in Lemma 9 with $\alpha=9 / 2$ yields

$$
\sum_{N=2}^{\infty} \mathbb{P}\left[\frac{\max _{1 \leq j \leq N} Y_{j}}{\sqrt{2 \log N}} \geq \frac{3}{2}\right]<\infty
$$

It now remains to obtain a further estimate on the second term on the right-hand side of (5.11).

To do this, for each $j=1, \ldots, k$ define the sequence of random variables

$$
U_{m}^{(j)}=\frac{Y_{j+m k}-\lambda^{k} Y_{j+(m-1) k}}{\sqrt{1-\lambda^{2 k}}} .
$$

Without loss of generality, let $l \neq m$ and notice that $\operatorname{Cov}\left(U_{l}^{(j)}, U_{m}^{(j)}\right)=0$, while $\operatorname{Var}\left(U_{m}^{j}\right)=1$ for all $m$ and $j$. Thus, for each $j=1, \ldots, k,\left\{U_{m}^{(j)}\right\}_{m=1}^{\infty}$ is an independent and identically distributed sequence of standard normal random variables. Further define $V_{m}^{(j)}=\max _{1 \leq l \leq m} U_{l}^{(j)}$, and $a_{N}=\mu \sqrt{2 \log N}$, where $\mu$ is given by (5.10). Then, by (5.10), the independence of $\left(U_{n}^{(1)}\right)_{n \geq 1}$, and the fact that all of the random variables $U_{n}^{(1)}$ are standard normal random variables, we get

$$
\begin{aligned}
\mathbb{P}\left[\frac{\max _{1 \leq j \leq n k} Y_{j+k}-\lambda^{k} Y_{j}}{\left.\sqrt{2 \log N} \leq 1-\varepsilon+\frac{3}{2} \lambda^{k}\right]}\right. & =\mathbb{P}\left[\max _{1 \leq j \leq k} V_{n}^{(j)} \leq a_{N}\right] \\
& \leq \mathbb{P}\left[V_{n}^{(1)} \leq a_{N}\right]=\Phi\left(a_{N}\right)^{n} \\
& \leq \Phi\left(a_{N}\right)^{\frac{N}{k}-1} .
\end{aligned}
$$

We now merely need to show that $\Phi\left(a_{N}\right)^{\frac{N}{k}-1}$ is summable over $N$. To do this, observe that since $a_{N} \rightarrow \infty$ and $\Phi\left(a_{N}\right) \rightarrow 1$ as $N \rightarrow \infty,(5.1)$ and L'Hôpital's rule yield

$$
\lim _{N \rightarrow \infty} \frac{\log \Phi\left(a_{N}\right)^{N / k-1}}{\left(\frac{N}{k}-1\right) \frac{1}{\sqrt{2 \pi}} \frac{1}{a_{N}} e^{-a_{N}^{2} / 2}}=\lim _{N \rightarrow \infty} \frac{\log \Phi\left(a_{N}\right)}{1-\Phi\left(a_{N}\right)} \frac{1-\Phi\left(a_{N}\right)}{\frac{1}{\sqrt{2 \pi}} \frac{1}{a_{N}} e^{-a_{N}^{2} / 2}}=-1 .
$$

Copyright $@$ by SIAM. Unauthorized reproduction of this article is prohibited. 
Hence

$$
\lim _{N \rightarrow \infty} \frac{\log \Phi\left(a_{N}\right)^{N / k-1}}{\frac{1}{k \mu \sqrt{2 \pi}} \frac{1}{\sqrt{2 \log N}} N^{1-\mu^{2}}}=-1,
$$

and therefore, as $\mu \in(0,1)$,

$$
\sum_{N=2}^{\infty} \Phi\left(a_{N}\right)^{N / k-1}<\infty
$$

Therefore, by (5.11), (5.12), (5.13), and (5.14), we get

$$
\sum_{N=1}^{\infty} \mathbb{P}\left[\max _{1 \leq j \leq N} Y_{j+k} \leq(1-\varepsilon) \sqrt{2 \log N}\right]<\infty
$$

The stationarity of the sequence now proves the assertion.

Acknowledgments. We are delighted to thank both anonymous referees for their careful and scholarly review of our original manuscript. Their comments were instrumental in correcting some errors in the proofs and in clarifying and improving the presentation throughout. We also thank the reviewers for pointing out and querying the relationship between the work in this paper and results both in the theory of nonstationary Gaussian processes and the theory of abstract stochastic evolution equations. These comments have motivated the inclusion of section 3.2 in this article.

\section{REFERENCES}

[1] V. Anh And A. Inoue, Financial markets with memory. I. Dynamic models, Stoch. Anal. Appl., 23 (2005), pp. 275-300.

[2] V. Anh, A. Inoue, AND Y. Kasahara, Financial markets with memory. II. Innovation processes and expected utility maximization, Stoch. Anal. Appl., 23 (2005), pp. 301-328.

[3] J. A. D. Appleby, I. Győri, And D. W. Reynolds, On exact rates of decay of solutions of linear systems of Volterra equations with delay, J. Math. Anal. Appl., 320 (2006), pp. 5677.

[4] J. A. D. Appleby, X. Mao, And M. Riedle, Geometric Brownian motion with delay: Mean square characterisation, Proc. Amer. Math. Soc., 137 (2009), pp. 339-348.

[5] J. A. D. Appleby, X. MaO, And H. Wu, On the size of the largest pathwise deviations of stochastic functional differential equations, Nonlinear Stud., to appear.

[6] J. A. D. Appleby, M. Riedle, And C. Swords, A Black-Scholes model with delay, submitted.

[7] J. A. D. Appleby AND H. Wu, Solutions of stochastic differential equations obeying the law of the iterated logarithm, with applications to financial markets, Electron. J. Probab., 14 (2009), pp. 912-959.

[8] J. A. D. Appleby And H. Wu, Exponential growth and Gaussian-like fluctuations of solutions of stochastic differential equations with maximum functionals, J. Phys. Conf. Ser., 138 (2008), 012002.

[9] M. Arriojas, Y. Hu, S.-E. Mohammed, and G. Pap, A delayed Black and Scholes formula, Stoch. Anal. Appl., 25 (2007), pp. 471-492.

[10] S. Blythe, X. MaO, And A. Shah, Razumikhin-type theorems on stability of stochastic neural networks with delays, Stochastic Anal. Appl., 19 (2001), pp. 85-101.

[11] T. Bollerslev, Generalized autoregressive conditional heteroskedasticity, J. Econometrics, 31 (1986), pp. 307-327.

[12] J.-P. Bouchaud And R. Cont, A Langevin approach to stock market fluctuations and crashes, Eur. Phys. J. B Condens. Matter Phys., 6 (1998), pp. 543-550.

[13] P. J. Brockwell, Representations of continuous-time ARMA processes. Stochastic methods and their applications, J. Appl. Probab., 41A (2004), pp. 375-382.

[14] P. J. Brockwell and R. A. Davis, Time Series: Theory and Methods, 2nd ed., SpringerVerlag, New York, 1991. 
[15] P. Brockwell and T. Marquardt, Lévy-driven and fractionally integrated ARMA processes with continuous time parameter, Statist. Sinica, 15 (2005), pp. 477-494.

[16] T. A. Burton and W. E. Mahfoud, Stability criterion for Volterra equations, Trans. Amer. Math. Soc., 279 (1983), pp. 143-174.

[17] T. Caraballo, I. D. Chueshov, P. Marín-Rubio, and J. Real, Existence and asymptotic behaviour for stochastic heat equations with multiplicative noise in materials with memory, Discrete Contin. Dyn. Syst., 18 (2007), pp. 253-270.

[18] G. J. Chen, F. C. Kong, And Z. Y. Lin, Answers to some questions about increments of a Wiener process, Ann. Probab., 14 (1986), pp. 1252-1261.

[19] Y. K. Chol AND J.-H. ChOI, On lag increments of a Gaussian process, Commun. Korean Math. Soc., 15 (2000), pp. 379-390.

[20] Y. K. ChOI AND K. S. Hwang, How big are the lag increments of a Gaussian process?, Comput. Math. Appl., 40 (2000), pp. 911-919.

[21] M. CsőRgo, Z.-Y. Lin, AND Q.-M. ShaO, On moduli of continuity for local times of Gaussian processes, Stochastic Process. Appl., 58 (1995), pp. 1-21.

[22] G. Da Prato And J. Zabczyk, Stochastic Equations in Infinite Dimensions, Cambridge University Press, Cambridge, UK, 1992.

[23] C. M. Deo, Some limit theorems for maxima of absolute values of Gaussian sequences, Sankhyā Ser. A, 34 (1972), pp. 289-292.

[24] C. M. Deo, Some limit theorems for maxima of nonstationary Gaussian processes, Ann. Statist., 1 (1973), pp. 981-984.

[25] R. Deo, C. Hurvich, And Y. Lu, Forecasting realized volatility using a long-memory stochastic volatility model: Estimation, prediction and seasonal adjustment, J. Econometrics, 131 (2006), pp. 29-58.

[26] O. Diekmann, S. A. van Gils, S. M. Verduyn Lunel, and H.-O. Walther, Delay Equations. Functional, Complex, and Nonlinear Analysis, Springer-Verlag, New York, 1995.

[27] A. D. Drozdov and V. B. Kolmanovskĭ, Stochastic stability of viscoelastic bars, Stochastic Anal. Appl., 10 (1992), pp. 265-276.

[28] J. Franke, W. K. Härdle, And C. M. Hafner, Statistics of Financial Markets: An Introduction, 2nd ed., Springer-Verlag, Berlin, 2008.

[29] J. K. Hale and S. M. Verduyn Lunel, Introduction to Functional Differential Equations, Springer-Verlag, New York, 1993.

[30] D. Hobson And L. C. G. Rogers, Complete models with stochastic volatility, Math. Finance, 8 (1998), pp. 27-48.

[31] K. Itô And H. P. McKean, Diffusion Processes and Their Sample Paths, 2nd ed., SpringerVerlag, Berlin, 1974.

[32] I. Karatzas and S. E. Shreve, Brownian Motion and Stochastic Calculus, 2nd ed., SpringerVerlag, New York, 1991.

[33] V. Kolmanovskil And A. MYshKis, Introduction to the Theory and Applications of Functional Differential Equations, Kluwer Academic Publishers, Dordrecht, The Netherlands, 1999.

[34] U. KüChler AND B. Mensch, Langevin's stochastic differential equation extended by a timedelayed term, Stochastics Stochastics Rep., 40 (1992), pp. 23-42.

[35] M. Leadbetter, G. Lindgren, and H. Rootzén, Extremes and Related Properties of Random Sequences and Processes, Springer Ser. Statist., Springer-Verlag, New York, 1983.

[36] K. LiU, Stationary solutions of retarded Ornstein-Uhlenbeck processes in Hilbert spaces, Statist. Probab. Lett., 78 (2008), pp. 1775-1783.

[37] K. LIU, Stochastic retarded evolution equations: Green functions, convolutions and solutions, Stoch. Anal. Appl., 26 (2008), pp. 624-650.

[38] X. Mao, Exponential Stability of Stochastic Differential Equations, Dekker, New York, 1994.

[39] X. MAO, Stochastic Differential Equations and Their Applications, Horwood, Chichester, 1997.

[40] X. MAO, Stochastic versions of the LaSalle theorem, J. Differential Equations, 153 (1999), pp. $175-195$.

[41] X. MaO, Delay population dynamics and environmental noise, Stoch. Dyn., 5 (2005), pp. 149162 .

[42] X. MaO And M. J. Rassias, Khasminskii-type theorems for stochastic differential delay equations, Stoch. Anal. Appl., 23 (2005), pp. 1045-1069.

[43] X. MaO And M. J. Rassias, Almost sure asymptotic estimations for solutions of stochastic differential delay equations, Int. J. Appl. Math. Stat., 9 (2007), pp. 95-109.

[44] M. B. Marcus, Upper bounds for the asymptotic maxima of continuous Gaussian processes, Ann. Math. Statist., 43 (1972), pp. 522-533.

[45] T. Marquardt and R. Stelzer, Multivariate CARMA processes, Stochastic Process. Appl., 117 (2007), pp. 96-120.

Copyright $@$ by SIAM. Unauthorized reproduction of this article is prohibited. 
[46] V. J. Mizel And V. Trutzer, Stochastic hereditary equations: Existence and asymptotic stability, J. Integral Equations, 7 (1984), pp. 1-72.

[47] V. J. Mizel and V. Trutzer, Asymptotic stability for stochastic hereditary equations, in Physical Mathematics and Nonlinear Partial Differential Equations (Morgantown, WV, 1983), Lecture Notes in Pure and Appl. Math. 102, Dekker, New York, 1985, pp. 57-70.

[48] S.-E. A. Mohammed, Stochastic Functional Differential Equations, Res. Notes in Math. 99, Pitman, Boston, 1984.

[49] D. Monrad And H. RootzÉn, Small values of Gaussian processes and functional laws of the iterated logarithm, Probab. Theory Related Fields, 101 (1995), pp. 173-192.

[50] M. Мотоо, Proof of the law of iterated logarithm through diffusion equation, Ann. Inst. Statist. Math., 10 (1958), pp. 21-28.

[51] J. PickAndS, III, Maxima of stationary Gaussian processes, Z. Wahrscheinlichkeitstheorie und Verw. Gebiete, 7 (1967), pp. 190-223.

[52] P. E. Protter, Stochastic Integration and Differential Equations, 2nd ed., Springer-Verlag, New York, 2004.

[53] M. Reiss, M. Riedle, and O. van GaAns, Delay differential equations driven by Lévy processes: Stationarity and Feller properties, Stochastic Process. Appl., 116 (2006), pp. 14091432.

[54] M. Reiss, M. Riedle, AND O. van GaAns, On Emery's inequality and a variation-of-constants formula, Stoch. Anal. Appl., 25 (2007), pp. 353-379.

[55] M. Riedle and J. M. A. M. van Neerven, A semigroup approach to stochastic delay equations in spaces of continuous functions, Semigroup Forum, 74 (2007), pp. 227-239.

[56] W. S. WANG AND L.-X. Zhang, Chung-type Law of the Iterated Logarithm on l $^{p}$-valued Gaussian processes, Acta Math. Sin. (Engl. Ser.), 22 (2006), pp. 551-560.

[57] L.-X. Zhang, On the fractal nature of increments of $l^{p}$-valued Gaussian processes, Stochastic Process. Appl., 71 (1997), pp. 91-110.

Copyright $@$ by SIAM. Unauthorized reproduction of this article is prohibited. 